\title{
Radiation Effects in Concrete for Nuclear Power Plants - Part I: Quantification of Radiation Exposure and Radiation Effects
}

\author{
K.G. Field ${ }^{\mathrm{a}, *}$, I. Remec ${ }^{\mathrm{a}}$, Y. Le Pape ${ }^{\mathrm{a}}$ \\ ${ }^{a}$ Oak Ridge National Laboratory, One Bethel Valley Road, Oak Ridge, TN 37831-6148
}

\begin{abstract}
A large fraction of light water reactor (LWR) construction utilizes concrete, including safetyrelated structures such as the biological shielding and containment building. Concrete is an inherently complex material, with the properties of concrete structures changing over their lifetime due to the intrinsic nature of concrete and influences from local environment. As concrete structures within LWRs age, the total neutron fluence exposure of the components, in particular the biological shield, can increase to levels where deleterious effects are introduced as a result of neutron irradiation. This work summarizes the current state of the art on irradiated concrete, including a review of the current literature and estimates the total neutron fluence expected in biological shields in typical LWR configurations. It was found a first-order mechanism for loss of mechanical properties of irradiated concrete is due to radiation-induced swelling of aggregates, which leads to volumetric expansion of the concrete. This phenomena is estimated to occur near the end of life of biological shield components in LWRs based on calculations of estimated peak neutron fluence in the shield after 80 years of operation.
\end{abstract}

*Corresponding author: fieldkg@ornl.gov (K.G. Field)

Preprint submitted to Nuclear Engineering and Design

September 26, 2014

(C) 2014. This manuscript version is made available under the Elsevier user license http://www.elsevier.com/open-access/userlicense/1.0/ 


\title{
Radiation Effects in Concrete for Nuclear Power Plants - Part I: Quantification of Radiation Exposure and Radiation Effects
}

\author{
K.G. Field ${ }^{\mathrm{a}, *}$, I. Remec ${ }^{\mathrm{a}}$, Y. Le Pape ${ }^{\mathrm{a}}$ \\ ${ }^{a}$ Oak Ridge National Laboratory, One Bethel Valley Road, Oak Ridge, TN 37831-6148
}

Keywords: concrete, irradiation, biological shield, swelling, amorphization

\section{Introduction}

Concrete is the most common and predominant material used in construction of commercial light water reactors (LWRs). Concrete-based LWR structures provide functions including foundation, support, shielding, and containment (Naus, 2012). Many of these structures are large and irreplaceable sections; therefore, the longevity of a specific plant could reside on the durability of these concrete structures. The microstructure and hence properties of concrete change overtime due to slow hydration, crystallization of amorphous constituents, and reactions between cement paste and aggregates as well as influences from the local environment such as humidity, temperature, radiation exposure, and/or chemical attack. As a concrete-based structure ages the deleterious effects due to the local environment are predicted to increase (Naus, 2012).

The influence of many environmental factors on large civil structures, similar to those in LWRs, such as chronic high-temperature exposure, freeze-thaw, and chemical attack has been studied in detail (e.g., Neville, 1963; Naus, 2005; Metha and Monteiro, 2005; Swamy, 1996). However, a LWR-specific environmental factor, the effect of radiation on the microstructure and properties of concrete-based structures, requires further investigation (Graves et al., 2013). The extension of the operating lifetimes of commercial LWRs has reignited the study of radiation effects on concrete. Of particular interest are the expected radiation exposure (fluence or dose) on concrete structures in typical LWR configurations and the resulting changes in mechanical and physical properties, and the mechanisms for such changes.

Several authors have reviewed irradiation effects in concrete, including Clark (1958), Komarovskii (1961), and Fillmore (2004) to name a few, but the most widely cited reference is the comprehensive compilation of work by Hilsdorf et al. (1978). The Hilsdorf compilation showed an inflection point towards a loss in the residual strength versus neutron fluence near $1.0 \times 10^{19} \mathrm{n} / \mathrm{cm}^{2}$, with similar effects observed for tensile strength. Kontani et al. (2010) evaluated the work cited in the Hilsdorf compilation and suggested much of the data might not be relevant to LWR concrete structures, as many of the irradiation experiments conducted at high dose $\left(>1.0 \times 10^{19} \mathrm{n} / \mathrm{cm}^{2}\right)$ were also conducted at temperatures above $100{ }^{\circ} \mathrm{C}$ and utilized concrete specimens atypical from LWR concrete. Maruyama et al. (2013) evaluated the changes in compressive strength with increasing neutron fluence and confirmed

\footnotetext{
${ }^{*}$ Corresponding author: fieldkg@ornl.gov (K.G. Field)
} 
a trend similar to that proposed by Hilsdorf et al. Given this, the evaluation of any concrete database is quite complex; many variables must be considered including the cement and aggregate, mix proportions, curing, environmental conditions during the experiment, and internal moisture content, and many of the reviews did not fully consider the inherent complexity of irradiated concrete. Furthermore, the previously cited studies, either by the original authors or through review papers, hinted at possible first-order mechanism(s) for degradation of irradiated concrete, but no final conclusions have yet been drawn. Finally, the reported fluence levels where the deleterious effects of radiation on concrete were observed were not considered in the context of the total fluence level expected for concrete structures in LWRs during long-term operation (>40 years), as the feasibility of LWRs life extensions was not thought to be an option at the time of publication.

This work seeks to evaluate the current understanding of irradiated concrete by (i) evaluating the expected radiation levels seen by concrete structures up to 80 years of life in LWRs, (ii) extending the study by Hilsdorf et al. to include data not previously reviewed in open literature, (iii) outlining the possible mechanisms for irreversible damage induced in irradiated concrete structures, and (iv) applying the current state of knowledge to develop a micromechanical model for understanding the interactions between the cement paste and aggregate in the presence of irradiation. Objectives i-iii will be covered within this paper while a recap of Objective iii and extensive investigation of Objective iv are the subjects of a companion paper (Le Pape et al., 2014).

\section{Biological Shield Radiation Exposure}

As noted, concrete is used in a wide range of structures for typical LWRs. A summary of concrete structures in LWR plants can be found in Hookham (1995). In relation to total radiation exposure, the biological shield walls are of upmost interest as these safety-related structures encase the reactor and provide essential radiation protection. The biological shield is the concrete structure in closest proximity to the reactor core and is expected to see the highest levels of radiation exposure over the lifetime of a LWR.

The concrete typically used in nuclear-safety-related structures, such as the biological shield, consists of Type II Portland cement, fine aggregates (e.g., sand), water, various mineral or chemical admixtures for improving properties or performance of the concrete, and either normal-weight or heavyweight coarse aggregate. For biological radiation shielding, heavyweight or dense aggregate materials such as barytes, limonites, magnetites, and ilmenites may have been used to reduce the section thickness and meet attenuation requirements. The thickness of a biological shield can reach a few meters in depth, depending on the design.

Radiation fields potentially affecting shield materials are typically described in terms of neutron fluence and gamma-ray dose. Hilsdorf et al. suggested that neutron fluence exceeding $1.0 \times 10^{19} \mathrm{n} / \mathrm{cm}^{2}$ may have a detrimental effect on concrete strength and elastic modulus. Kontani et al. $(2010,2013)$ suggested the effects of neutron and gamma radiation can be subdivided into the effects on water/hydrated phases and crystalline phases, with gamma radiation having little effect on crystalline phases at doses relevant to LWR applications but a potential effect on hydrated phases. With respect to neutron interactions, their work acknowledged a potential significant effect neutron irradiation could have on the crystalline phases in the paste and aggregate. Furthermore, initial gamma-ray experiments conducted by Kontani et al. (2013) indicated limited water decomposition solely by gamma-ray exposure and suggested little effect on the strength of concrete. Based on these observations, 
and the suggestion by Hilsdorf et al., this work focuses on the detrimental effects of neutron exposure to irradiated concrete with limited discussions on gamma-ray doses.

The expected fluence levels and neutron fluence limit before concrete degradation remain a matter of discussion as $1.0 \times 10^{20} \mathrm{n} / \mathrm{cm}^{2}$ for fast neutrons and $2.0 \times 10^{10} \mathrm{rad}$ for gamma irradiation has been suggested by Maruyama et al. (2013) as threshold levels, while ACI Committee 349 (2002) cites the Hilsdorf compilation and suggests $1.0 \times 10^{21} \mathrm{n} / \mathrm{cm}^{2}$ is where loss of mechanical properties are observed and places a conservative fluence limit at $1.0 \times 10^{13} \mathrm{n} / \mathrm{cm}^{2}$ to prevent lifetime radiation induced degradation, and British Standards Institute 4975 (1990) states neutron radiation should not have a significant effect below $5.0 \times 10^{17} \mathrm{n} / \mathrm{cm}^{2}$. Therefore, from the perspective of this work and research on the life extension of nuclear power plants, it is critical to assess a possible biological shield total radiation exposure of up to 80 years and what, if any, effect the total neutron fluence will have on the properties of concrete. While there is no routine monitoring of radiation fields in the biological shields of nuclear power plants, valuable information can be obtained from reactor pressure vessel (RPV) surveillance programs and used to infer information on the radiation fields in biological shields. Many nuclear power plants have deployed dosimetry measurements outside the RPV, providing data on radiation doses observed outside the RPV and near the surface of the biological shield. In the following sections, initial work on extrapolating the data from the RPV surveillance reports to characterizing the radiation field of biological shields is discussed.

\subsection{Radiation Transport Simulations}

The radiation transport simulations were based on computer models developed previously for the benchmarking of the radiation transport calculation methodology for the LWRs RPV surveillance programs and for the investigations of the RPV neutron exposure (Remec and Kam, 1997; Remec, 1999).

The transport calculations were performed using the DORT computer code from the DOORS3.2a code system, which includes one-, two- and three-dimensional (1-D, 2-D, and 3D) discrete-ordinates neutron and photon transport codes (ORNL, 1997). The flux synthesis method was used, which relies on 2-D and 1-D transport calculations to obtain an estimation of the neutron fluxes in the 3-D geometries. One 2-D calculation models the horizontal cross section of the reactor in the $\mathrm{r}-\vartheta$ geometry. It is used to compute the variations of the neutron field in the radial direction (which is the main direction of the neutron transport from the core toward the RPV and beyond) and in the azimuthal direction. The second calculation is a 2-D calculation in cylindrical $\mathrm{r}-\mathrm{z}$ geometry, in which the reactor core is modeled as a finite-height cylinder. The third calculation is made for the 1-D (r) cylindrical model of the reactor. The $\mathrm{r}-\mathrm{z}$ and $1-\mathrm{D} \mathrm{r}-$ calculations are combined to obtain the axial variations of the neutron field. The synthesis method remains the de facto state-of-the-art method used in the nuclear industry for the calculations of neutron fluxes for RPV surveillance programs. However, three-dimensional radiation transport codes are available and could be used in future analysis (Rhoades, 1997; Evans et al., 2010).

The neutron and gamma-ray cross-sections were taken from the latest of the BUGLEseries libraries, namely, the BUGLE-B7, which is a 47-neutron and 20-gamma-ray multigroup library based on ENDF/B-VII.0 nuclear data (Risner et al., 2011). The P3 approximations to the angular dependence of the anisotropic scattering cross sections (i.e., the P0 to P3 Legendre components) were taken into account, and a symmetric S8 "directional quadrature set" (i.e., a set of discrete directions and angular quadratures) was used for all transport calculations. 
The simulations provided multigroup neutron and gamma-ray fluxes throughout the reactor, RPV, and the biological shield, which were used to determine neutron fluxes, dose rates, heating rates, and reaction rates. Good agreement, typically within $10 \%$, was observed between the calculated reaction rates and reaction rates measured in the cavity between the RPV and biological shield for the neutron threshold dosimeters ${ }^{238} \mathrm{U}(\mathrm{n}, \mathrm{f}),{ }^{58} \mathrm{Ni}(\mathrm{n}, \mathrm{p})$, ${ }^{54} \mathrm{Fe}(\mathrm{n}, \mathrm{p}),{ }^{46} \mathrm{Ti}(\mathrm{n}, \mathrm{p}),{ }^{63} \mathrm{Cu}(\mathrm{n}, \alpha)$, therefore, confirming the validity of the calculations; however, no measurements with gamma-ray sensitive monitors were available for benchmarking.

Pressurized water reactors (PWRs) typically exhibit higher radiation fields outside the RPV than boiling water reactors (BWRs) (Lucius Pitkin, Inc., 2013). Among the PWR designs, the highest radiation levels outside the RPV are typically observed in two-loop plants followed by lower levels in three-loops plants and at the lowest levels are four-loop plants (Lucius Pitkin, Inc., 2013). Based on these observations, the transport calculations were based on plants designed by Westinghouse, with one plant having a three-loop configuration and the other a two-loop configuration. Design characteristics of each plant are listed in Table 1. The concrete compositions and density assumed for the two different plant designs are given in Table 2. The composition for the 3-loop PWR is based on 02-B ordinary concrete (Remec and Kam, 1997; Maerker, 1987) while the 2-loop PWR is based on a NBS type 04 concrete (White et al., 1994). For the three loop plant, the higher iron content is due to assuming a $0.7 \%$ volume addition of ferrous rebar (Remec and Kam, 1997).

Several limitations are inherent in this approach. Both plants analyzed were of Westinghouse design; therefore, the results obtained could be representative for plants of similar design but may be considerably different for plants designed by either Combustion Engineering or Babcock \& Wilcox. In addition, for both plants, the neutron source was prepared from the fuel-cycle specific core power and burn-up distributions and both plants utilized low-leakage fuel loading patterns in which partially burned fuel assemblies are loaded on the core periphery. Even for a plant of similar design, different fuel loading patterns may result in substantial changes in neutron and gamma-ray fields outside the RPV. Finally, the attenuation of neutrons through the concrete shield is affected by density and composition of concrete and only two concretes were investigated. It is probable that other plants will have different compositions than the ones investigated here and furthermore the water content of a specific biological shield will vary with time. Therefore, the results reported here should be viewed as examples of the possible expected values for a very specific reactor design and not as definitive limiting values for the fleet of currently operating LWRs.

Table 1: Selected parameters of the two PWRs analyzed.

\begin{tabular}{ccccc}
\hline Type & $\begin{array}{c}\text { Thermal } \\
\text { Power }(\mathrm{MW})\end{array}$ & $\begin{array}{c}\text { RPV } \\
\text { Thickness }\end{array}$ & $\begin{array}{c}\text { Thickness of } \\
\text { Reactor } \\
\text { Cavity }\end{array}$ & $\begin{array}{c}\text { Inner Radius } \\
\text { of Biological } \\
\text { Shield }\end{array}$ \\
\hline \hline 3-loop PWR & 2300 & $24.2 \mathrm{~cm}$ & $17.1 \mathrm{~cm}$ & $238.8 \mathrm{~cm}$ \\
\hline 2-loop PWR & 1876 & $16.8 \mathrm{~cm}$ & $16.0 \mathrm{~cm}$ & $200.6 \mathrm{~cm}$ \\
\hline
\end{tabular}


Table 2: Density and chemical compositions of biological shields used in radiation transport simulations from Remec and Kam (1997) and White et al. (1994). Compositions are given in weight percent.

\begin{tabular}{ccccccccccccccc}
\hline Type & $\begin{array}{c}\text { Density } \\
\left(\mathrm{g} / \mathrm{cm}^{3}\right)\end{array}$ & $\mathrm{H}$ & $\mathrm{C}$ & $\mathrm{O}$ & $\mathrm{Na}$ & $\mathrm{Mg}$ & $\mathrm{Al}$ & $\mathrm{Si}$ & $\mathrm{K}$ & $\mathrm{Ca}$ & $\mathrm{Fe}$ \\
\hline \hline 3-loop PWR & 2.28 & 0.51 & 0.10 & 50.50 & 1.62 & 0.22 & 3.43 & 34.09 & 1.31 & 4.40 & 3.82 \\
\hline 2-loop PWR & 2.31 & 0.57 & 0.10 & 50.70 & 1.74 & 0.26 & 4.66 & 32.11 & 1.96 & 6.64 & 1.26 \\
\hline
\end{tabular}

\subsection{Results and Discussion Based on Radiation Transport Simulations}

Computed neutron flux variation through the RPV, cavity, and the biological shield in the radial direction for the three-loop plant is shown in Figure 1. The curves are shown for neutron fluxes with different cut-off energies, as well as the thermal neutron flux $(\mathrm{E}<0.41$ $\mathrm{eV})$ to emphasize that different energy regions of neutron spectrum behave differently and consequently the neutron spectrum changes considerably through the structures considered. The calculations estimate the neutron fluxes (for the $0.1 \mathrm{MeV}$ and $1 \mathrm{MeV}$ cut-off) observed at the biological shield face are 20-30\% lower than the maximum flux at the RPV outer radius. Based on these observations, a conservative estimate for the fluxes in concrete biological shields can be inferred from the maximum fast neutron flux $(\mathrm{E}>1 \mathrm{MeV})$ reported in RPV surveillance reports of individual LWRs. Results indicated the neutron spectra in the cavity region of the two-loop and three-loop PWRs are similar but that the two-loop plant has two to four times higher neutron and gamma fluxes than the three-loop plant. Using the data from Figure 1 and data for the two-loop plant, estimated neutron fluences for 40, 60 and 80 years of operation are given in Table 2, assuming a 92\% capacity factor for the 2-loop and 3-loop PWRs. 


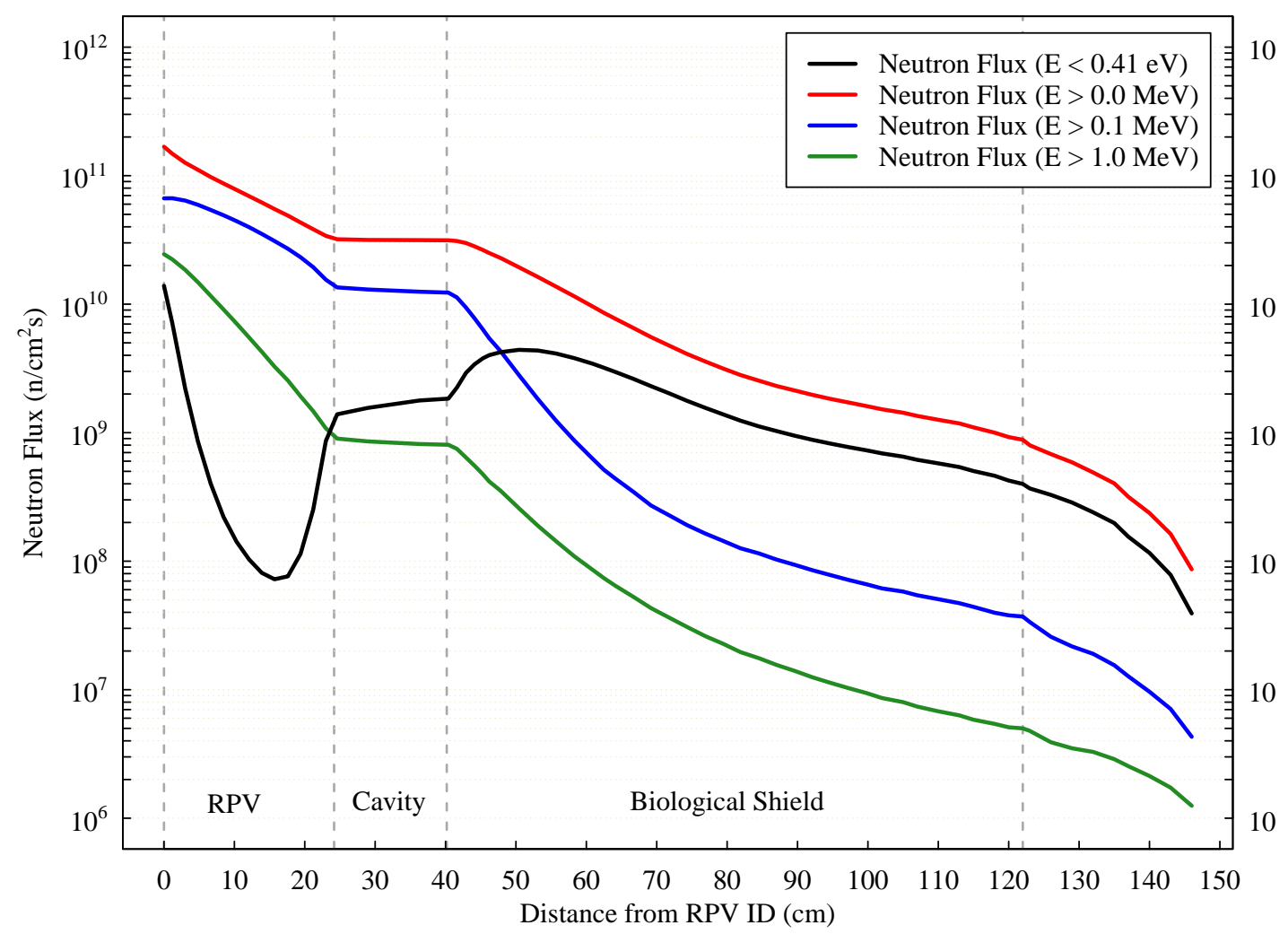

Figure 1: Distribution of neutron flux for given neutron energy cut-offs in a three-loop PWR in the radial direction from the core.

As discussed in later sections, information about the neutron flux energy distribution (spectrum) for the irradiated concrete studies in the literature varies. Often only a qualitative description is provided. The following interpretations are assumed: "slow" indicates the thermal neutrons with energies up to a few eV dominate the neutron spectrum; a component of higher energy neutron flux was likely present, but it was not included in the reported value and, if taken into account, would not increase significantly (i.e., less than $~ 30 \%$ ) the value of the neutron fluence reported. "Fast" indicates the fluence of neutrons with energies above $0.1 \mathrm{MeV}, 1.0 \mathrm{MeV}$, or, in rare cases, some intermediate value. Fast neutrons are always accompanied by lower energy neutrons, the latter due to either streaming in from the surroundings of concrete specimens, or inevitably being produced by slowing down of the energetic neutrons in the material. The ratio of slow-to-fast neutrons can vary considerably based on the irradiation location and detailed local configuration of the irradiation capsule and concrete specimens. Since such detailed information is typically not provided for the experiments in the literature, the renormalization from fast to total neutron fluence is not possible. However, the change from fast to total neutron fluence is very significant: the fast fluence value reported could be associated with the total fluence value anywhere from the actually reported (fast) fluence value up to 40 times or even higher total neutron fluence values. 
Table 3: Estimated neutron fluence for plant configurations analyzed at 40, 60, and 80 years of operation assuming a $92 \%$ capacity factor.

\begin{tabular}{ccccc}
\hline \multirow{2}{*}{ Years of Operation } & \multirow{2}{*}{ PWR Type } & \multicolumn{3}{c}{ Neutron Fluence $\left(\mathrm{n} / \mathrm{cm}^{2}\right)$} \\
\cline { 3 - 5 } & & $\mathrm{E}>0.0 \mathrm{MeV}$ & $\mathrm{E}>0.1 \mathrm{MeV}$ & $\mathrm{E}>1.0 \mathrm{MeV}$ \\
\hline \hline \multirow{2}{*}{40} & 2-loop & $7.3 \times 10^{19}$ & $2.8 \times 10^{19}$ & $3.2 \times 10^{18}$ \\
\cline { 2 - 5 } & 3-loop & $3.6 \times 10^{19}$ & $1.3 \times 10^{19}$ & $8.7 \times 10^{17}$ \\
\hline \multirow{2}{*}{60} & 2-loop & $1.1 \times 10^{20}$ & $4.2 \times 10^{19}$ & $4.9 \times 10^{18}$ \\
\hline \multirow{2}{*}{80} & 3-loop & $5.4 \times 10^{19}$ & $2.0 \times 10^{19}$ & $1.3 \times 10^{18}$ \\
\hline & 2-loop & $1.5 \times 10^{20}$ & $5.6 \times 10^{19}$ & $6.5 \times 10^{18}$ \\
\hline & 3-loop & $7.2 \times 10^{19}$ & $2.6 \times 10^{19}$ & $1.7 \times 10^{18}$ \\
\hline
\end{tabular}

Based on the available experimental data in the Hilsdorf compilation, there is no conclusive evidence that fast neutrons produce more radiation damage in concrete than slow neutrons (Kaplan, 1983). Therefore, Table 3 includes the neutron fluences with energies above $1.0 \mathrm{MeV}$ (i.e.: $\phi_{E>1.0 \mathrm{MeV}}=\int_{1.0 \mathrm{MeV}}^{20 \mathrm{MeV}} \phi(E) d E$ where $E$ is the neutron energy and $\phi$ is the neutron fluence), $0.1 \mathrm{MeV}$ (i.e.: $\phi_{E>0.1 \mathrm{MeV}}=\int_{0.1 \mathrm{MeV}}^{20 \mathrm{MeV}} \phi(E) d E$ ), and the integral neutron fluence (i.e.: $\phi_{E>0.0 \mathrm{MeV}}=\int_{0.0 \mathrm{MeV}}^{20 \mathrm{MeV}} \phi(E) d E$ ). For the purpose of this paper the lower energy boundary in the integral is defined as the neutron fluence energy cutoff. The importance of the neutron fluence energy cutoff is paramount. For the $1 \mathrm{MeV}$ energy cutoff, the two plants considered remain below the fluence $1.0 \times 10^{19} \mathrm{n} / \mathrm{cm}^{2}$ throughout the 40 year design lifetime and the extended operation to 80 years. For the energy cutoff of $0.1 \mathrm{MeV}$, both plants barely exceed the $1.0 \times 10^{19} \mathrm{n} / \mathrm{cm}^{2}$ fluence at 40 years and reach up to $\sim 6.0 \times 10^{19}$ $\mathrm{n} / \mathrm{cm}^{2}$ at 80 years of operation. On the other hand, if integral neutron flux is considered, including the contribution from thermal neutrons, both plants exceed the $1.0 \times 10^{19} \mathrm{n} / \mathrm{cm}^{2}$ fluence at 40 years of operation and reach fluences in excess of $1.0 \times 10^{20} \mathrm{n} / \mathrm{cm}^{2}$ at 80 years of operation. Determining which energy cutoff, if any, is correct for the fluence determination is crucial for the assessment of the concrete degradation, in particular for the operation during extended plant life.

Another aspect of interest is the geometrical extent of the region in which the damage in concrete may occur. Figure 1 shows the neutron flux profile drops off as a function of radial distance from the core vertical axis. Inside the concrete of the biological shield the flux above 1.0 MeV and above $0.1 \mathrm{MeV}$ decrease by about one order of magnitude over a distance of 25 $\mathrm{cm}$. The flux of thermal neutrons behaves differently and actually reaches a local maximum at $\sim 9 \mathrm{~cm}$ inside the concrete due to the increased moderation in concrete. However, the total neutron flux decreases inside the concrete, albeit at a slower rate than the more energetic components. This suggests that although biological shields will see neutron fluences within the range where deleterious effects are expected, the effects would be restricted to a layer of concrete near the surface facing the pressure vessel. It should also be noted that no information is available on the effects of neutron flux (fluence rate) on the microstructure or mechanical properties of irradiated concrete, and therefore, it still remains a question of whether accelerated testing such as that summarized in following sections can be used 
to extrapolate the degradation effects to lower fluxes observed in the biological shields of LWRs.

The overall importance of the neutron fluence cutoff energy and the lack of concise information on fluence cutoff used in the experiments reported in the literature indicate a need for further research on the deleterious effects of neutron irradiation on concrete. Initial steps towards this understanding were taken by reevaluating literature cited by Hilsdorf et al. and data published after the time of the Hilsdorf compilation, as described below.

\section{Effects of Neutron Radiation on Mechanical Properties of Concrete}

\subsection{Sources of Additional Data}

Current understanding of the effects of radiation on the mechanical properties of concrete is based on the so-called Hilsdorf curve(s), which was based on data published in the literature in the late 1970s. More recent work by Kontani et al. (2013) revised the Hilsdorf compressive strength curve and revealed that the residual compressive strength data after neutron exposure above $5.0 \times 10^{19} \mathrm{n} / \mathrm{cm}^{2}$ were not representative of concrete used or radiation fields seen in LWRs. This review and other derivative reviews were focused on data presented by Hilsdorf et al. with limited attention given to extracting data from works cited by Hilsdorf et al. or investigating new data not presented in the Hilsdorf compilation. Acknowledging that important data may not have been in the public domain when the Hilsdorf report was completed, and expecting that more experiments were performed since that time, an expanded literature search was conducted here with the objective to expand the database and to attempt to identify possible first-order deleterious effects in irradiated concrete.

Table 4 presents a summary of the literature utilized in the following sections. An inherent difficulty with the current literature is a lack of systematic studies spanning the entire fluence range to be expected within the biological shield of LWRs. Only a few authors, Crispino et al. (1971); Dubrovskii et al. (1966a,b, 1968); Elleuch et al. (1971, 1972); Granata and Montagnini (1971); Rappeneau et al. (1966); Dubois et al. (1969) and Rockwell (1948) conducted studies to fluences higher than $1 \times 10^{19} \mathrm{n} / \mathrm{cm}^{2}$, although the neutron energy cutoff varies among the studies, and few works cited a neutron spectrum, allowing for complete normalization of the neutron fluence across all datasets. For those studies where both fast and slow neutron fluences were reported, the fast neutron spectrum is reported here and shown in Table 4.

Similar to the limited information on neutron energy, many studies did not provide detailed information on the aggregates and the aggregate type and only a simple description was provided. For example, Seeberger and Hilsdorf (1982) listed the chemical composition of the limestone aggregates used but Gray (1971)/Kelly et al. (1969) did not. Limestone consists primarily of calcite $\left(\mathrm{CaCO}_{3}\right)$ with additions of dolomite and minor additions of quartz or other siliceous minerals and the varying degree of these additions changes depending on the geographical location where the limestone was extracted for the experiment. Therefore, its probable the limestones used between Seeberger and Hilsdorf (1982) and Gray (1971)/Kelly et al. (1969) varied significantly but can not be directly confirmed. Here, the description provided by the original authors is utilized but it must be recognized that significant variation is expected within a single classified aggregate subsection, such as limestone, in Table 4.

Furthermore, a significant portion of the studies were conducted at test temperatures $>100{ }^{\circ} \mathrm{C}$. Conducting experiments above $100{ }^{\circ} \mathrm{C}$ will result in loss of all evaporable, strongly 
absorbed, and possibly chemically combined water from the cement paste given sufficient exposure times and temperatures, resulting in paste dehydration and a different concrete microstructure from irradiations conducted below $100{ }^{\circ} \mathrm{C}$ (Naus, 2010). A reason for such high irradiation temperatures in many of the studies was that they focused on gas-cooled reactor applications where the expected concrete temperatures exceeded those of commercial LWRs. Another possible reason is that the higher neutron flux needed to obtain high fluences necessitated the irradiation of samples in environments (locations) where sample heating was increased. These limitations were taken into consideration during the analysis of the presented data in the following sections and discussed in more detail in Section 5. For the purpose of this paper, the work of Dubois et al. (1969) is excluded from further analysis as this study was conducted in a $\mathrm{CO}_{2}$ atmosphere, resulting in carbonation of samples and therefore skewed results compared to the rest of the database in which irradiations were conducted in air or the presence of a non-reactive gas such as helium. 
Table 4: Summary of original research published on neutron irradiated concrete and mortars.

\begin{tabular}{|c|c|c|c|c|c|c|c|c|c|}
\hline Ref. & $\begin{array}{l}\text { Neutron } \\
\text { Fluence }\end{array}$ & $\begin{array}{l}\text { Energy } \\
\text { Cut-off }\end{array}$ & $\begin{array}{l}\text { Temp. } \\
\text { Range } \\
\left({ }^{\circ} \mathrm{C}\right)\end{array}$ & Aggregates & Cement Type & $\begin{array}{l}\text { A/C } \\
\text { Ratio }\end{array}$ & $\begin{array}{l}\mathrm{W} / \mathrm{C} \\
\text { Ratio }\end{array}$ & $\begin{array}{l}\text { Specimen } \\
\text { Geometry }\end{array}$ & $\begin{array}{l}\text { Reported } \\
\text { Values* }\end{array}$ \\
\hline $\begin{array}{c}\text { Alexander } \\
\text { (1963) }\end{array}$ & $\begin{array}{l}0.25 \times 10^{19}- \\
2.00 \times 10^{19}\end{array}$ & $\begin{array}{c}\text { slow \& } \\
\text { fast }\end{array}$ & $\begin{array}{l}20- \\
100\end{array}$ & $\begin{array}{c}\text { gravel, } \\
\text { limestone, } \\
\text { magnetite, } \\
\text { ilemnite, } \\
\text { granite, baryte, } \\
\text { slag, } \\
\text { whinestone, } \\
\text { firebrick }\end{array}$ & $\begin{array}{c}\text { ordinary } \\
\text { Portland cement } \\
\text { (OPC), high } \\
\text { alumina cement, } \\
\text { low-heat-slag } \\
\text { cement, super } \\
\text { sulphate } \\
\text { cement, OPC } \\
\text { with fuel ash }\end{array}$ & $\begin{array}{l}3.0 \\
6.0\end{array}$ & - & $\begin{array}{l}0.50 \mathrm{in} . \\
\text { cube, } 2 \mathrm{in} . \\
\text { cube, } 0.50 \\
\text { in. } \\
\text { cylinder, } 2 \\
\text { in. } \times 1.75 \\
\text { in. } \times 8 \mathrm{in} . \\
\text { beams }\end{array}$ & $\mathrm{W}, \mathrm{f}_{\mathrm{c}}, \mathrm{E}$ \\
\hline $\begin{array}{c}\text { Batten } \\
(1960) ; \\
\text { Price } \\
\text { et al. } \\
(1957)\end{array}$ & $\begin{array}{c}0.43 \times 10^{19}- \\
7.50 \times 10^{19}\end{array}$ & thermal & 50 & river sand & $\begin{array}{c}\text { Portland } \\
\text { cement, Cement } \\
\text { Fondu }\end{array}$ & $\begin{array}{l}3.0 \\
8.0\end{array}$ & $\begin{array}{l}0.45 \\
0.50\end{array}$ & $\begin{array}{l}2 \text { in. } \times 2 \\
\text { in. } \times 8 \text { in. } \\
\text { beams }\end{array}$ & $\mathrm{W}, \mathrm{f}_{\mathrm{c}}, \mathrm{f}_{\mathrm{t}}$ \\
\hline $\begin{array}{c}\text { Blosser } \\
\text { et al. } \\
(1958) ; \\
\text { Rockwell } \\
(1948)\end{array}$ & $\begin{array}{l}1.00 \times 10^{9}- \\
1.00 \times 10^{17}\end{array}$ & thermal & $10-37$ & $\begin{array}{l}\text { rock, sand or } \\
\text { baryte, haydite }\end{array}$ & Portland cement & 4.5 & 0.61 & - & $f_{C}$ \\
\hline $\begin{array}{l}\text { Christiani } \\
\text { et al. } \\
(1971) ; \\
\text { Granata } \\
\text { and } \\
\text { Mon- } \\
\text { tagnini } \\
(1971)\end{array}$ & $\begin{array}{l}2.50 \times 10^{18}- \\
3.80 \times 10^{18}\end{array}$ & fast & $\begin{array}{l}100- \\
125\end{array}$ & $\begin{array}{c}\text { limestone, } \\
\text { baryte }\end{array}$ & Portland cement & 3.0 & 0.50 & $\begin{array}{l}4 \mathrm{~cm} \times 4 \\
\mathrm{~cm} \times 16 \\
\mathrm{~cm} \text { beams }\end{array}$ & $D, \rho, f_{t}, E$ \\
\hline $\begin{array}{l}\text { Crispino } \\
\text { et al. } \\
(1971)\end{array}$ & $\begin{array}{l}1.00 \times 10^{19}- \\
1.00 \times 10^{20}\end{array}$ & thermal & $\begin{array}{c}130- \\
280\end{array}$ & $\begin{array}{c}\text { limestone, } \\
\text { baryte }\end{array}$ & Portland cement & - & - & $\begin{array}{l}4 \mathrm{~cm} \times 4 \\
\mathrm{~cm} \times 16 \\
\mathrm{~cm} \text { beams }\end{array}$ & $\mathrm{D}, \mathrm{P}$ \\
\hline $\begin{array}{c}\text { Dickeman } \\
(1951)^{1}\end{array}$ & $1.30 \times 10^{18}$ & Integrated & 120 & limonite & $\begin{array}{l}\text { Portland } \\
\text { Cement }\end{array}$ & 6.4 & 0.39 & - & $\mathrm{D}, \mathrm{f}_{\mathrm{C}}$ \\
\hline $\begin{array}{l}\text { Dubois } \\
\text { et al. } \\
(1969)^{1}\end{array}$ & $\begin{array}{c}5.00 \times 10^{18}- \\
1.50 \times 10^{19}\end{array}$ & $\begin{array}{c}\mathrm{E}>1.0 \\
\mathrm{MeV}\end{array}$ & $\begin{array}{l}170- \\
280\end{array}$ & $\begin{array}{l}\text { expanded clay, } \\
\text { vilmolithe }\end{array}$ & $\begin{array}{l}\text { aluminous } \\
\text { cement }\end{array}$ & 2.4 & $\begin{array}{l}0.60 \\
0.44\end{array}$ & $\begin{array}{c}2.5 \mathrm{~cm} \mathrm{x} \\
2.5 \mathrm{~cm} \times 10 \\
\mathrm{~cm} \text { beams, } \\
4 \mathrm{~cm} \mathrm{x} 4 \\
\mathrm{~cm} \mathrm{x} 16 \\
\mathrm{~cm} \text { beams }\end{array}$ & $\begin{array}{c}D, w, f_{c} \\
f_{t}, E\end{array}$ \\
\hline
\end{tabular}




\begin{tabular}{|c|c|c|c|c|c|c|c|c|c|}
\hline Ref. & $\begin{array}{l}\text { Neutron } \\
\text { Fluence }\end{array}$ & $\begin{array}{l}\text { Energy } \\
\text { Cut-off }\end{array}$ & $\begin{array}{l}\text { Temp. } \\
\text { Range } \\
\left({ }^{\circ} \mathrm{C}\right)\end{array}$ & Aggregates & Cement Type & $\begin{array}{l}\text { A/C } \\
\text { Ratio }\end{array}$ & $\begin{array}{l}\mathrm{W} / \mathrm{C} \\
\text { Ratio }\end{array}$ & $\begin{array}{l}\text { Specimen } \\
\text { Geometry }\end{array}$ & $\begin{array}{c}\text { Reported } \\
\text { Values* }\end{array}$ \\
\hline $\begin{array}{l}\text { Dubrovskii } \\
\text { et al. } \\
(1966 \mathrm{~b})\end{array}$ & $\begin{array}{l}2.00 \times 10^{21}- \\
2.40 \times 10^{21}\end{array}$ & $\begin{array}{c}\overline{\mathrm{E}}=0.23 \\
\mathrm{MeV}\end{array}$ & $\begin{array}{c}200- \\
550\end{array}$ & chromite & $\begin{array}{l}\text { Portland } \\
\text { cement, } \\
\text { water-glass }\end{array}$ & - & - & $\begin{array}{c}15 \mathrm{~mm} \mathrm{x} \\
15 \mathrm{~mm} \mathrm{OD}\end{array}$ & $\begin{array}{c}\mathrm{D}, \mathrm{W}, \mathrm{f}_{\mathrm{C}}, \\
\mathrm{E}\end{array}$ \\
\hline $\begin{array}{l}\text { Dubrovskii } \\
\text { et al. } \\
(1966 a)^{1}\end{array}$ & $\begin{array}{c}0.04 \times 10^{19}- \\
1.70 \times 10^{19}\end{array}$ & $\begin{array}{l}\mathrm{E}>0.8 \\
\mathrm{MeV}^{2}\end{array}$ & $\begin{array}{l}50- \\
350\end{array}$ & $\begin{array}{l}\text { river sand } \\
\text { (quartz) and } \\
\text { sandstone }\end{array}$ & Portland cement & 4.7 & 0.50 & $\begin{array}{c}40 \mathrm{~mm} \mathrm{x} \\
40 \mathrm{~mm} \mathrm{OD}\end{array}$ & $\begin{array}{c}D, \rho, W \\
f_{C}, E\end{array}$ \\
\hline $\begin{array}{l}\text { Dubrovskii } \\
\text { et al. } \\
(1968)^{1}\end{array}$ & $\begin{array}{l}1.30 \times 10^{21}- \\
1.70 \times 10^{21}\end{array}$ & Integrated & 350 & serpentine & - & - & - & - & $\mathrm{D}, \mathrm{f}_{\mathrm{C}}, \mathrm{E}$ \\
\hline $\begin{array}{l}\text { Dubrovskii } \\
\text { et al. } \\
(1970)^{1}\end{array}$ & $\begin{array}{l}0.40 \times 10^{19}- \\
5.50 \times 10^{19}\end{array}$ & $\begin{array}{c}\mathrm{E}>0.8 \\
\mathrm{MeV}\end{array}$ & $\begin{array}{c}100- \\
400\end{array}$ & hematite & Portland cement & 8.0 & 1.01 & $\begin{array}{c}15 \mathrm{~mm} \mathrm{x} \\
15 \mathrm{~mm} \mathrm{OD}\end{array}$ & $\begin{array}{c}\mathrm{D}, \mathrm{W}, \mathrm{f}_{\mathrm{C}}, \\
\mathrm{E}\end{array}$ \\
\hline $\begin{array}{c}\text { Elleuch } \\
\text { et al. } \\
(1971 \\
1972)\end{array}$ & $\begin{array}{l}1.20 \times 10^{19}- \\
1.11 \times 10^{20}\end{array}$ & $\begin{array}{c}\mathrm{E}>1.0 \\
\mathrm{MeV}\end{array}$ & $\begin{array}{c}150- \\
240\end{array}$ & serpentine & $\begin{array}{c}\text { Lafarge } \\
\text { aluminous } \\
\text { cement }\end{array}$ & 3.9 & 0.38 & $\begin{array}{c}2.5 \mathrm{~cm} \mathrm{x} \\
2.5 \mathrm{~cm} \times 5 \\
\mathrm{~cm} \text { beams, } \\
2.5 \mathrm{~cm} \mathrm{x} \\
2.5 \mathrm{~cm} \times 10 \\
\mathrm{~cm} \text { beams }\end{array}$ & $\begin{array}{c}D, W, f_{c}, \\
f_{t}, E\end{array}$ \\
\hline $\begin{array}{c}\text { Fujiwara } \\
\text { et al. } \\
(2009)^{1}\end{array}$ & $\begin{array}{c}0.70 \times 10^{18} \\
1.20 \times 10^{19}\end{array}$ & $\begin{array}{c}\mathrm{E}>0.1 \\
\mathrm{MeV}\end{array}$ & $50-56$ & - & - & 6.0 & 0.55 & $\begin{array}{l}100 \mathrm{~mm} \mathrm{x} \\
50 \mathrm{~mm} \mathrm{OD}\end{array}$ & $\begin{array}{c}\mathrm{D}, \mathrm{W}, \mathrm{f}_{\mathrm{C}}, \\
\mathrm{E}\end{array}$ \\
\hline $\begin{array}{c}\text { Gray } \\
(1971) ; \\
\text { Kelly } \\
\text { et al. } \\
(1969)\end{array}$ & $\begin{array}{l}1.20 \times 10^{18}- \\
4.33 \times 10^{19}\end{array}$ & fast $^{2}$ & 55 & flint, limestone & Portland cement & 2.7 & 0.36 & $\begin{array}{l}.25 \text { in. } x \\
0.50 \text { in. } \\
\text { OD }\end{array}$ & $\begin{array}{c}D, \rho, W \\
f_{t}, E\end{array}$ \\
\hline $\begin{array}{l}\text { Halliday } \\
(1956)^{1}\end{array}$ & $3.16 \times 10^{19}$ & - & - & - & - & - & - & - & - \\
\hline $\begin{array}{c}\text { Houben } \\
(1969) ; \\
\text { Van de } \\
\text { Schaaf } \\
(1967 \\
1969)\end{array}$ & $\begin{array}{l}3.00 \times 10^{19}- \\
8.00 \times 10^{19}\end{array}$ & fast & $\begin{array}{l}150- \\
200\end{array}$ & $\begin{array}{c}\text { baryte, } \\
\text { magnetite, } \\
\text { hollith }\end{array}$ & $\begin{array}{c}\text { Portland } \\
\text { cement, HOC }\end{array}$ & 5.0 & $\begin{array}{l}0.14- \\
0.16\end{array}$ & $\begin{array}{c}8 \mathrm{~mm} \times 8 \\
\mathrm{~mm} \times 70 \\
\mathrm{~mm} \text { beams }\end{array}$ & $f_{t}, E$ \\
\hline $\begin{array}{l}\text { Idei } \\
\text { et al. } \\
\text { (1990) }\end{array}$ & $\begin{array}{l}2.07 \times 10^{11}- \\
1.86 \times 10^{17}\end{array}$ & $\begin{array}{c}\mathrm{E}>0.1 \\
\mathrm{MeV}\end{array}$ & $<100$ & river rock & Portland cement & 6.5 & 0.48 & - & $f_{c}, f_{t}, E$ \\
\hline $\begin{array}{c}\text { Lyon } \\
(1951)^{1}\end{array}$ & $\begin{array}{l}2.06 \times 10^{18}- \\
4.40 \times 10^{18}\end{array}$ & Integrated & - & heavy aggregate & $\mathrm{MgO}$ cement & 22.0 & 1.62 & - & $\mathrm{f}_{\mathrm{C}}$ \\
\hline
\end{tabular}




\begin{tabular}{|c|c|c|c|c|c|c|c|c|c|}
\hline Ref. & $\begin{array}{l}\text { Neutron } \\
\text { Fluence }\end{array}$ & $\begin{array}{l}\text { Energy } \\
\text { Cut-off }\end{array}$ & $\begin{array}{l}\text { Temp. } \\
\text { Range } \\
\left({ }^{\circ} \mathrm{C}\right)\end{array}$ & Aggregates & Cement Type & $\begin{array}{l}\text { A/C } \\
\text { Ratio }\end{array}$ & $\begin{array}{l}\text { W/C } \\
\text { Ratio }\end{array}$ & $\begin{array}{l}\text { Specimen } \\
\text { Geometry }\end{array}$ & $\begin{array}{c}\text { Reported } \\
\text { Values* }\end{array}$ \\
\hline $\begin{array}{l}\text { Pederson } \\
\text { (1971) }\end{array}$ & $\begin{array}{l}8.50 \times 10^{18}- \\
3.00 \times 10^{19}\end{array}$ & fast & $<80$ & quartz & Portland cement & 3.0 & 0.40 & $\begin{array}{c}11.3 \mathrm{~mm} \mathrm{x} \\
11.3 \mathrm{~mm} \\
\text { OD }\end{array}$ & $\begin{array}{c}\mathrm{D}, \mathrm{W}, \mathrm{f}_{\mathrm{C}}, \\
\mathrm{E}\end{array}$ \\
\hline $\begin{array}{l}\text { Rappeneau } \\
\text { et al. } \\
(1966)^{1}\end{array}$ & $\begin{array}{c}1.40 \times 10^{19}- \\
1.10 \times 10^{20}\end{array}$ & fast & $\begin{array}{l}130- \\
260\end{array}$ & $\begin{array}{c}\text { serpentine, } \\
\text { corundum, rare } \\
\text { earths }\end{array}$ & $\begin{array}{c}\text { Lafarge } \\
\text { aluminous } \\
\text { cement }\end{array}$ & 4.1 & 0.38 & $\begin{array}{c}4 \mathrm{~cm} \times 4 \\
\mathrm{~cm} \times 16 \mathrm{~cm} \\
\text { beams, } 2.5 \\
\mathrm{~cm} \times 2.5 \\
\mathrm{~cm} \times 10 \\
\mathrm{~cm} \text { beams }\end{array}$ & $\begin{array}{c}D, W, f_{C}, \\
f_{t}, E\end{array}$ \\
\hline $\begin{array}{c}\text { Rockwell } \\
(1956)^{1}\end{array}$ & $3.00 \times 10^{18}$ & thermal & - & - & - & - & - & - & - \\
\hline $\begin{array}{l}\text { Seeberger } \\
\text { and } \\
\text { Hilsdorf } \\
(1982)^{1}\end{array}$ & $\begin{array}{c}2.50 \times 10^{17}- \\
5.00 \times 10^{18}\end{array}$ & fast & $<150$ & $\begin{array}{l}\text { limestone, } \\
\text { quartizite }\end{array}$ & Portland cement & 3.0 & 0.50 & $\begin{array}{c}32 \mathrm{~mm} \mathrm{x} \\
16 \mathrm{~mm} \mathrm{OD}\end{array}$ & $f_{C}, E$ \\
\hline $\begin{array}{l}\text { Stoces } \\
\text { et al. } \\
(1970)\end{array}$ & $\begin{array}{c}3.00 \times 10^{16}- \\
4.20 \times 10^{18}\end{array}$ & $\begin{array}{l}\mathrm{E}>0.1 \\
\mathrm{MeV}^{2}\end{array}$ & $20-80$ & gravel, sand & Portland cement & 3.6 & 0.35 & cylinder & $\begin{array}{c}\mathrm{D}, \mathrm{W}, \mathrm{f}_{\mathrm{C}}, \\
\mathrm{E}\end{array}$ \\
\hline
\end{tabular}

${ }^{1}$ Not cited in Hilsdorf et al. (1978)

2 Neutron energy spectrum reported

*Symbols: W - weight change, D - dimensional change, $\rho$ - density change,

$f_{C}$ - compressive strength, $f_{t}$ - tensile strength, E - elastic modulus

\subsection{Irradiated Concrete Compressive Strength}

As shown in Table 3, a majority of the available literature reported residual compressive strength values for neutron-irradiated concrete. Figure 2 presents the data set within the neutron fluence range of $1 \times 10^{14} \mathrm{n} / \mathrm{cm}^{2}$ to $1 \times 10^{22} \mathrm{n} / \mathrm{cm}^{2}$, while Figure 3 presents the data within the range of $1 \times 10^{18} \mathrm{n} / \mathrm{cm}^{2}$ to $1 \times 10^{21} \mathrm{n} / \mathrm{cm}^{2}$. The dashed line in the Figures 2-3 and subsequent figures represent the nonlinear least-square estimate of an exponential function as a guide to the readers eye while the shaded polygon represents the $90 \%$ prediction interval, unless otherwise noted. Compressive strength data at lower fluence levels than those shown in Figure 2 are reported in literature but showed insignificant changes in the compressive strength after neutron radiation and therefore are not presented. The updated data in Figures 2 and 3 agree with the downward sloping trend in the relative compressive strength with increasing neutron fluence presented by Hilsdorf et al. (1978) and Maruyama et al. (2013). Here, the decrease in compressive strength is most pronounced at fluence values greater than $1.0-2.0 \times 10^{19} \mathrm{n} / \mathrm{cm}^{2}$ where the residual compressive strength is lower bounded by about $50 \%$ of the initial strength at about $1.0 \times 10^{20} \mathrm{n} / \mathrm{cm}^{2}$. It should be noted the neutron energy cutoff of these data points vary from study to study. 


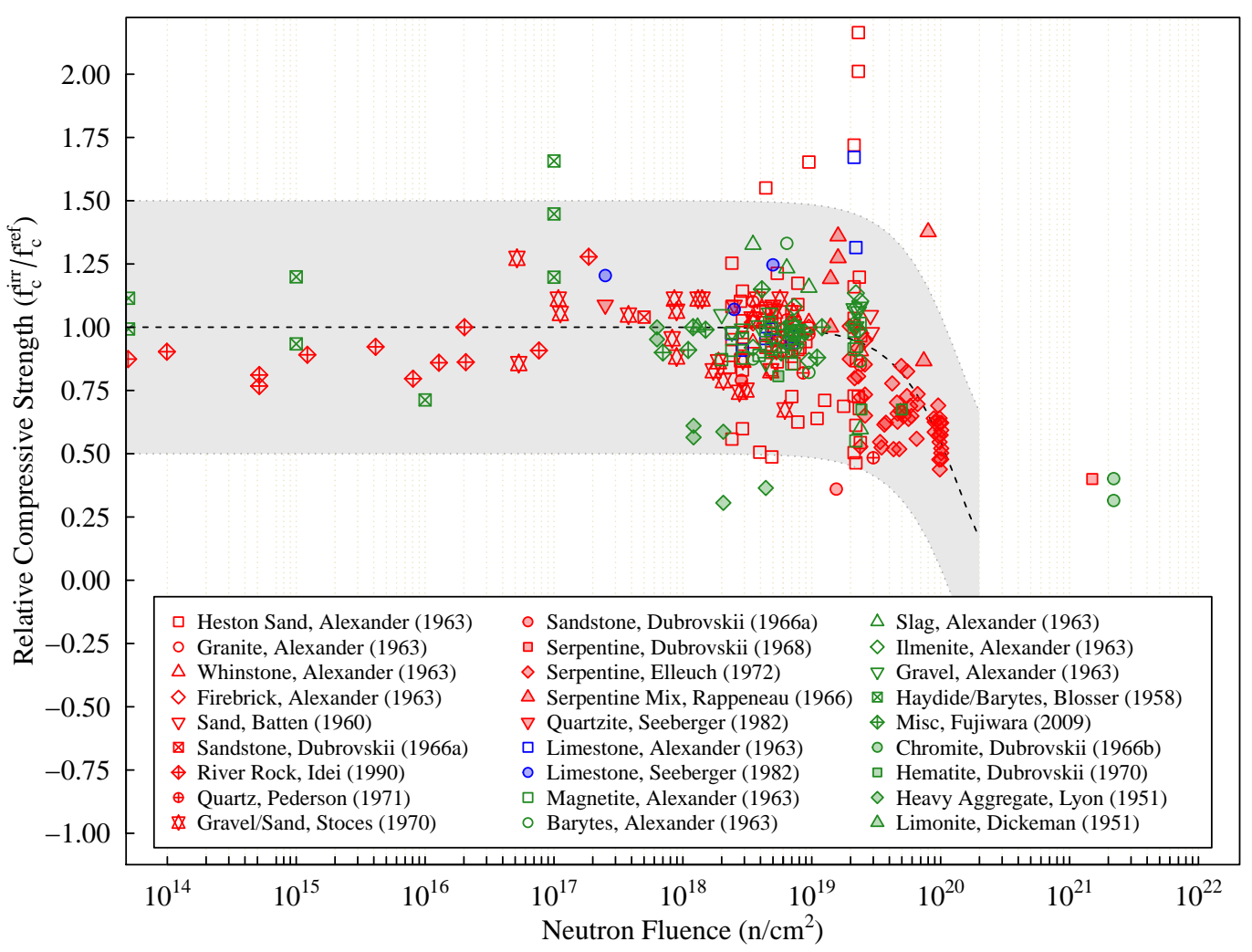

Figure 2: Relative compressive strength of concrete and mortar specimens versus neutron fluence. The neutron spectrum and specimen temperature vary between experiments. Siliceous concrete is depicted with red symbols, calcareous with blue, and miscellaneous concretes with green. Filled symbols indicate experiments conducted above $100{ }^{\circ} \mathrm{C}$; open symbols indicate experiments conduced below $100{ }^{\circ} \mathrm{C}$. Mix design can be determined by cross referencing with Table 3 . A decrease in compressive strength above $2 \times 10^{19} \mathrm{n} / \mathrm{cm}^{2}$ is suggested. 


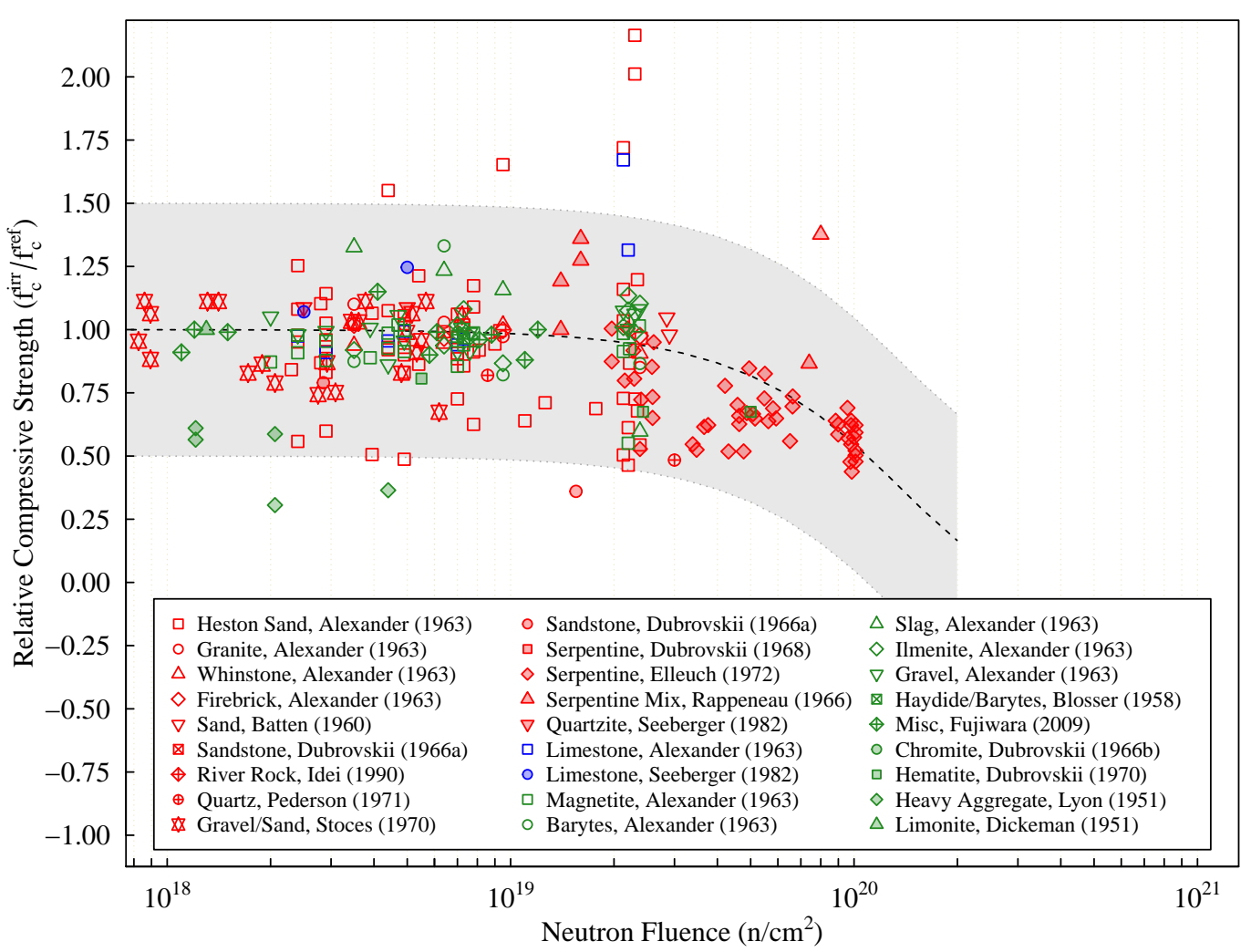

Figure 3: Relative compressive strength of concrete and mortar specimens versus neutron fluence in the range of $1 \times 10^{18} \mathrm{n} / \mathrm{cm}^{2}$ to $1 \times 10^{21} \mathrm{n} / \mathrm{cm}^{2}$ from Figure 2. The neutron spectrum and specimen temperature vary between experiments. Siliceous concrete is depicted with red symbols, calcareous with blue, and miscellaneous concretes with green. Filled symbols indicate experiments conducted above $100{ }^{\circ} \mathrm{C}$; open symbols indicate experiments conduced below $100{ }^{\circ} \mathrm{C}$. Mix design can be determined by cross referencing with Table 3. A decrease in compressive strength above $2 \times 10^{19} \mathrm{n} / \mathrm{cm}^{2}$ is suggested.

A data partitioning scheme is proposed to assist in analyzing mechanical and physical property data for irradiated concretes. A significant fraction of the studies in Table 3 utilized Portland cement but the aggregate type, water-to-cement ratio, and other mix design parameters varied greatly from study to study, and hence the reported trends is completed based on the relative compressive strength $\left(f_{c}^{i r r} / f_{c}^{r e f}\right)$. The compressive strength of concrete is based on (i) the quality of the hardened cement paste, that is, limiting the paste porosity by lowering the initial water-to-cement ratio (Feret, 1892), and (ii) the limitation of the average distance between the aggregate by obtaining the highest packing ratio of the aggregate skeleton (de Larrard and Belloc, 1997). For ordinary concrete, de Larrard and Belloc found that the composite strength of concrete can be modeled using a simple relation:

$$
f_{c_{c}}^{\prime} \approx a \cdot f_{c_{m}}^{\prime}
$$

where $f_{c_{m}}^{\prime}$ is the strength of the hardened cement paste (the matrix), $f_{c_{c}}^{\prime}$ is the strength of concrete composite, and $a$ is an empirical coefficient function of the nature of the aggregate and its packing density. As discussed previously, neutrons are hypothesized to have 
little effect on altering the strength of the cement matrix (Kontani et al., 2013) due to its limited long range order compared to the aggregates. However, the strength of the matrix can potentially be altered by several other mechanisms which can be categorized as (i) intrinsic damage mechanisms of the cement paste, that is, thermal damage and gamma irradiation induced damage, and (ii) aggregate-induced damage mechanisms to the paste, that is, restrained deformation-induced cracking.

Aggregate-induced damage mechanisms to the paste can be caused by cement paste shrinkage, differential thermal expansion and/or irradiation-induced swelling. These mechanisms are discussed more thoroughly in the companion paper. Regarding the intrinsic mechanisms, Janotka and Nürnbergerová (2005) observed only minor modifications of the compressive strength of ordinary cement paste with $\sim 10 \%$ silica fume, $(\mathrm{w} / \mathrm{c}=0.32)$ exposed sequentially to $40{ }^{\circ} \mathrm{C}, 60{ }^{\circ} \mathrm{C}$ and $100{ }^{\circ} \mathrm{C}$ for 28 days for each temperature level, although evidence of shrinkage was noted. Komonen and Penttala (2003) exposed ordinary Portland cement paste up to temperatures of $1000{ }^{\circ} \mathrm{C}$ for 60 minutes and indicated degradation due to coarsening of the pore structure although up to $400{ }^{\circ} \mathrm{C}$ the residual compressive capacity of the cement paste was still over 70\%. Maruyama et al. (2014) conducted an extensive study to determine the effects of drying and heating (up to $90{ }^{\circ} \mathrm{C}$ ) for mortars and concretes and indicated a minimum value on the residual compressive capacity of $80 \%$ and linked such degradation of properties to both the intrinsic and aggregate-induced damage mechanisms. Given this, pure cement paste was not investigated making direct translations of the effect of intrinsic damage mechanisms difficult. These results, among others such as Pihlajavaara (1974), indicate the intrinsic damage mechanisms of the cement paste based on thermal exposure can have an impact on the strength of the matrix and needs to be considered to fully grasp the experimental data presented for irradiated concrete experiments.

In regards to the intrinsic effect due to gamma irradiation, Kontani et al. (2010) reviewed Hilsdorf's compilation and found that Sommers (1969), which provided data for the downward sloping trend with increasing gamma ray dose, was biased due to the leaching of concrete in deionized water. The remaining data are very limited in number (Alexander, 1963; Gray, 1971; Kelly et al., 1969) and did not present any particular trend on the compressive strength of concrete after gamma-ray irradiation. Gamma irradiation experiments on mortar bars by Soo and Milian (2001) are inherently difficult to interpret due to several time-dependent mechanisms occurring simultaneously (hydration, carbonation, and drying) and because their conclusions about the absence of a dose rate effect and dose received at a given time contradict Łowińska-Kluge and Piszora (2008) observations made by scanning electron microscopy.

Although the possibility of intrinsic degradation mechanisms in the cement paste should not be completely ruled out during irradiation experiments, the approach adopted here to simplify analysis is to assume that irradiated concrete strength is primarily dominated by the intrinsic properties of the aggregate and its interaction with the cement paste. This justification for partitioning the data based on the mineralogical characteristics of the aggregate is supported by the work of Seeberger and Hilsdorf (1982) and Gray (1971)/Kelly et al. (1969), which found the aggregate type strongly determines its neutron radiation tolerance. Here, a three-component partitioning scheme is proposed to assess these assumptions: those aggregates composed primarily of silicates, those primarily of carbonates, and others (or miscellaneous/trap), which were typically heavy aggregates like hematite or baryte used for shielding. Studies with no information on the aggregate composition used were classified as miscellaneous/trap. The application of such a classification scheme is based solely on the available information from the original articles and might be skewed, such as carbonate 
bearing aggregates could contain a significant fraction of siliceous phases embedded within.

Using the partitioning scheme, most data above $2.0 \times 10^{19} \mathrm{n} / \mathrm{cm}^{2}$ in Figures 2 and 3 corresponded to studies using siliceous concretes and mortars on which the downward sloping trend is based. Given this, several miscellaneous aggregate (hematite, chromite) data points seem to reinforce the proposed decreasing trend, although the perceived slope appears to be less for miscellaneous aggregates compared to silicate-based aggregates. This observation supports the hypothesis that the radiation tolerance of concrete and mortars may be material specific with an emphasis on the aggregate composition.

A majority of the studies conducted above $2.0 \times 10^{19} \mathrm{n} / \mathrm{cm}^{2}$, were at temperatures above $100{ }^{\circ} \mathrm{C}$ (filled circles). Only the studies of Alexander (1963), Batten (1960), and Pederson (1971) were conducted below $100{ }^{\circ} \mathrm{C}$ (open circles) and showed that relative strength ratios as low as 0.50 are possible at fluences near $2.0 \times 10^{19} \mathrm{n} / \mathrm{cm}^{2}$ (slow, thermal, and $\mathrm{E}$ $>0.1 \mathrm{MeV}$, respectively), further reinforcing the downward sloping trend above $2.0 \times 10^{19}$ $\mathrm{n} / \mathrm{cm}^{2}$ for neutron-irradiated concrete. Furthermore, several investigations conducted compression tests on specimens which were aged at room temperature (not plotted), aged at high temperature but not irradiated (not plotted), and irradiated at elevated temperatures. These studies found the difference in the decrease in strength between the aged at high temperature and high temperature irradiated specimens was larger than the bounds of experimental error, suggesting, to a certain degree, the significant loss in strength seen above $2.0 \times 10^{19} \mathrm{n} / \mathrm{cm}^{2}$ can be partially attributed to the detrimental effects of neutron irradiation on concrete.

For fluences below $1.0 \times 10^{19} \mathrm{n} / \mathrm{cm}^{2}$, it is difficult to conclude any effects of neutron irradiation on concrete specimens and it is not possible to distinguish specific behavior differentiating the various aggregates using the proposed classification scheme. The observed scatter at lower fluences, most likely due to limiting factors discussed later such as sub-sized specimens, ranges between $75 \%$ and $125 \%$ of the reference compressive strength. The only data that fall out of the suggested scatter band are the studies reported by Lyon (1951) on magnesia-bearing concrete where no temperature or detailed description for the irradiation test was reported.

\subsection{Irradiated Concrete Tensile Strength}

Figure 4 presents the compiled data on the residual tensile strength values for neutronirradiated concrete. For the purpose of evaluating the effects of radiation on concrete, the tensile strength may be more important than compressive strength as it directly relates to the structural performance of a component under shear loading. The reported data were obtained either by indirect tension tests such as the study conducted by Gray (1971)/Kelly et al. (1969) or using more common flexural tests (modulus of rupture). The modulus of rupture typically reports higher strength values compared to direct or in-direct tensile tests, and therefore the data in Figure 4 are normalized by the non-irradiated tensile strength data in an effort to remove bias in the data due to testing configuration. The same partitioning scheme used in Figures 2 and 3 is also used for Figure 4.

The influence of the neutron irradiation on the tensile strength of concrete is significantly greater than for compressive strength. Residual tensile strength at values near $2.0 \times 10^{19}$ $\mathrm{n} / \mathrm{cm}^{2}$ were lower bounded at $25 \%$ of the initial value compared to the $50 \%$ of the initial value seen in the compressive strength. The highest reported strength loss was reported for irradiations conducted above $100{ }^{\circ} \mathrm{C}$. This result is expected, as elevated temperature exposures of siliceous concrete have been reported to decrease the tensile strength with increasing temperature (Zhang et al., 2002). Given this, the decreases observed here (25\% 
of initial) are sustainably greater than those reported in the literature (50-90\% of initial) for concretes tested after elevated temperature. The substantial variation between the known effects of elevated temperature on the tensile strength of concrete and those reported for irradiated concrete in Figure 4 indicates, to a certain degree, that the observed losses in strength are primarily a result of radiation, with elevated irradiation temperatures having only a minor effect. This would indicate that concrete and mortars irradiated above $1.0 \times 10^{19}$ $\mathrm{n} / \mathrm{cm}^{2}$ using neutrons could see a marked decrease in structural performance while in service in a neutron radiation environment.

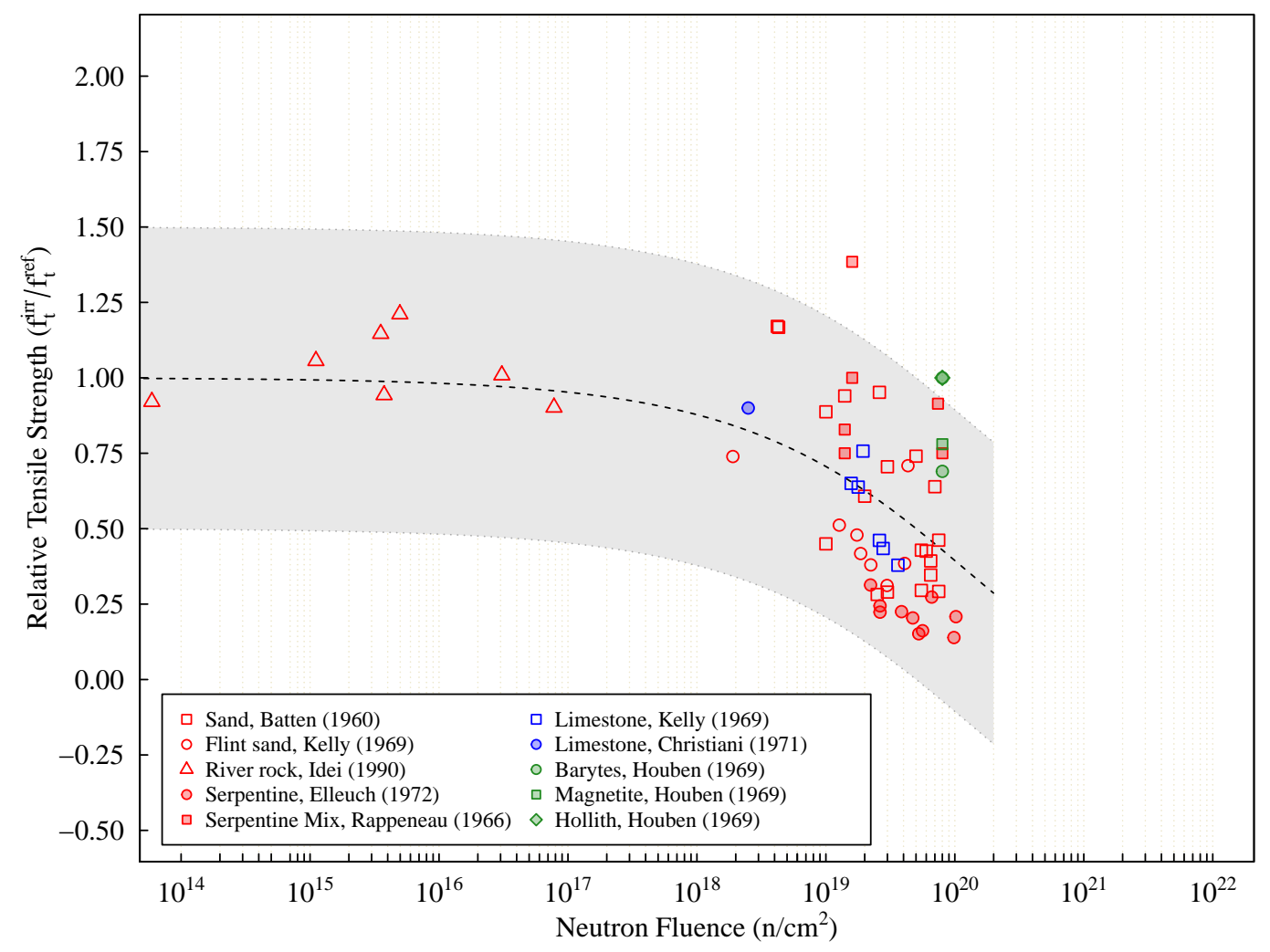

Figure 4: Relative tensile (flexural) strength values of concrete and mortar specimens versus neutron fluence. The neutron spectrum and the specimen temperature vary between experiments. Siliceous concrete is depicted with red symbols, calcareous with blue, and miscellaneous concretes with green. Filled symbols indicate experiments conducted above $100{ }^{\circ} \mathrm{C}$; open symbols indicate experiments conduced below $100{ }^{\circ} \mathrm{C}$. A strong decrease in tensile strength above $1 \times 10^{19} \mathrm{n} / \mathrm{cm}^{2}$ is suggested.

The variability and larger scatter in the tensile data compared to those of the compressive strength could be due to variances in the mix design such as aggregate size and mixture proportions as tensile strength has been shown to be sensitive to such variables (Naus, 2005). The limited data on irradiated tensile strength values limits a cross comparison between the three different aggregate types. Only two studies were conducted on a nonsilicate or carbonate material, with Houben $(1969) /$ Van de Schaaf $(1967,1969)$ reporting 
reductions between $15-30 \%$ of the initial strength on barytes and magnetite mortars while Rappeneau et al. (1966) reported a tensile strength increase under irradiation. The limited number of calcareous concretes showed trends similar to those of siliceous aggregate-based concretes, indicating a possible insensitivity of the tensile strength to the aggregate used in irradiated concrete, although further research would be needed to confirm.

\subsection{Irradiated Concrete Modulus of Elasticity}

The decrease in elastic modulus with neutron exposure is presented in Figure 5. Several different techniques were employed to obtain the elastic modulus including pulse velocity, tangent static modulus, and resonance frequency. The data here are normalized against the non-irradiated elastic modulus reported by the authors in an attempt to normalize the data across different experimental configurations.

Based on the reported data, the impact of neutron irradiation on the elastic modulus is less than that of compressive strength or tensile strength. A gradual decreasing trend is observed above $2.0 \times 10^{19} \mathrm{n} / \mathrm{cm}^{2}$ for concretes containing all aggregate types. The largest decrease in elastic modulus is for those studies conducted above $100{ }^{\circ} \mathrm{C}$ with a maximum loss of $\sim 60 \%$ reported by Dubrovskii et al. (1966a) on siliceous concrete to a fluence $>1.5 \times 10^{19}$ $\mathrm{n} / \mathrm{cm}^{2}(\mathrm{E}>0.8 \mathrm{MeV})$, while limited elastic modulus loss is reported for studies conducted below $100{ }^{\circ} \mathrm{C}$ at similar fluence levels.

As reported by Hilsdorf et al. (1978), the decrease in the modulus of elasticity with neutron fluence above $1.0 \times 10^{19} \mathrm{n} / \mathrm{cm}^{2}$ could be due to simultaneous high temperature and radiation exposure in the reported data. The effect of temperature on the modulus of elasticity of concrete has been reported to be monotonically decreasing with increasing temperature by several authors (Freskakis, 1980; Xiao and Konig, 2004). Furthermore, the decrease in elasticity is dependent on the type of aggregate as siliceous-bearing concrete is known to have greater decreases in elasticity than other aggregate types (Diererichs and Ehm, 1981). Based on these observations, the trend shown in Figure 4 could be a superposition of both the deleterious effect of neutron and high temperature exposure, or solely an artifact of the high-temperature during the irradiation studies. The companion article (Le Pape et al., 2014) develops a deconvolution method based on the evolution of the elastic properties of concrete and its individual constituents (cement paste and aggregate). This approach could only be applied to Gray (1971)/Kelly et al. (1969), and Elleuch et al. (1971, 1972) due to a lack of data on individual constituents in other referenced articles. This deconvolution method indicates, based on limited data, that indeed the response of the irradiated concrete modulus of elasticity is a superposition of both neutron irradiation and elevated temperature. 


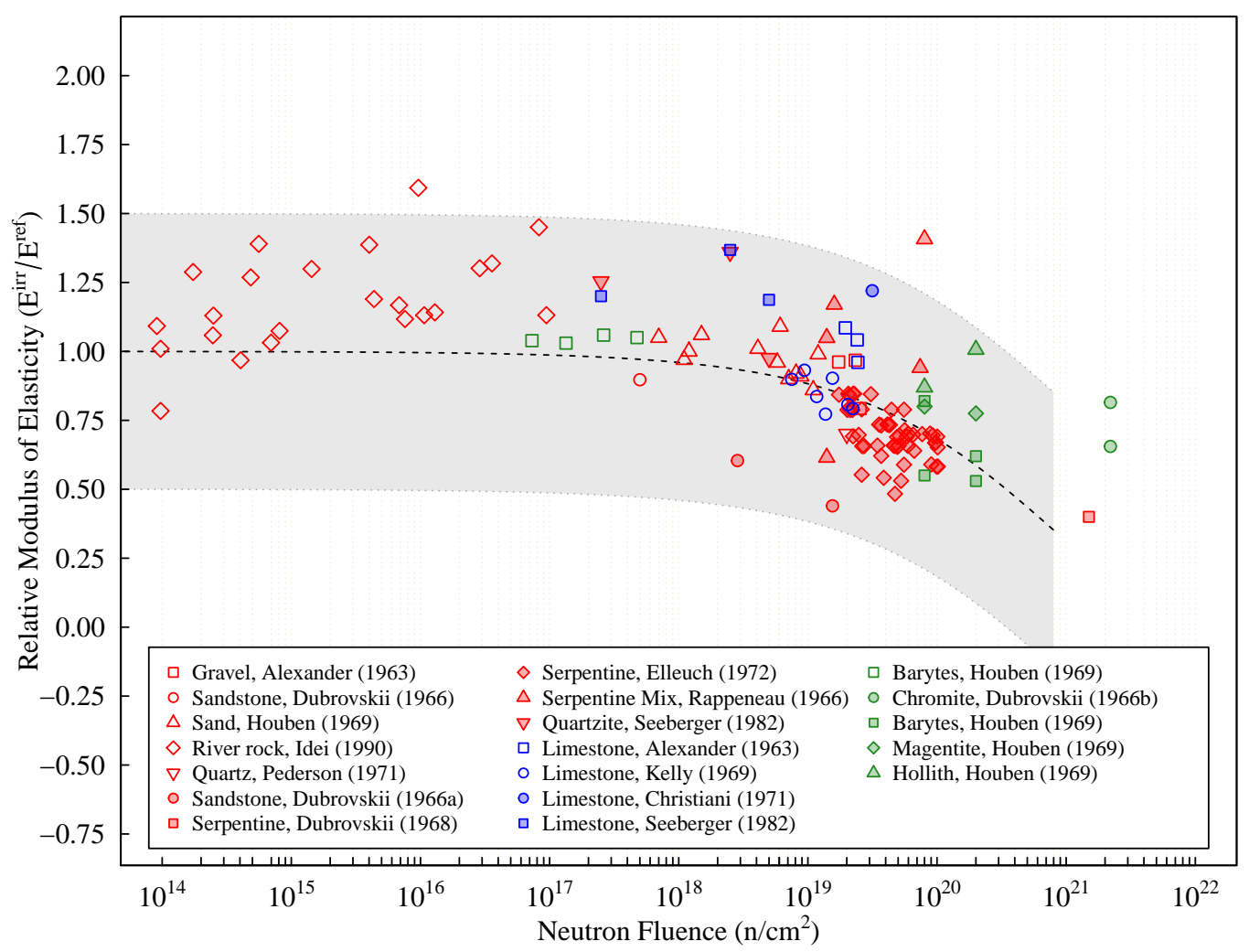

Figure 5: Relative elastic modulus values of concrete and mortar specimens versus neutron fluence. The neutron spectrum and the specimen temperature vary between experiments. Siliceous concrete is depicted with red symbols, calcareous with blue, and miscellaneous concretes with green. Filled symbols indicate experiments conducted above $100{ }^{\circ} \mathrm{C}$; open symbols indicate experiments conduced below $100{ }^{\circ} \mathrm{C}$. A gradual decrease in modulus of elasticity is suggested above $1 \times 10^{18} \mathrm{n} / \mathrm{cm}^{2}$.

\section{Effects of Radiation on the Physical Properties of Concrete}

\subsection{Irradiated Concrete Weight Loss}

The weight loss as a function of neutron exposure is presented in Figure 6. No specific trends were observed between different aggregate types, and a large amount of experimental scatter is observed. Several papers reported a weight gain (Alexander, 1963; Rappeneau et al., 1966), an anomaly which could be explained due to the irradiation environment or sample-handling procedures but could not be conclusively determined here.

The majority of studies that reported weight loss had a 7-8\% of free water by weight mix design and conducted either long-term aging in a fixed environment or pre-drying of the specimens prior to irradiation. For example, the study conducted by Elleuch et al. (1971, 1972) pre-dried concrete specimens at $250{ }^{\circ} \mathrm{C}$ prior to irradiation, followed by weighing of the specimens. The study of Gray (1971)/Kelly et al. (1969) indicated a $\sim 5 \%$ water by weight at the beginning of irradiation. As shown in Figure 6, the weight loss never exceeded 
$5 \%$ of the original weight of the irradiated concrete specimens, indicating the weight loss in the concrete specimens is most likely due to dehydration of cement paste.

Many authors cited in Figure 6 also conducted weight loss measurements on samples exposed to the same thermal-hydric history as the irradiated specimens and indicated analogous weight loss between the two sample sets. For example, Dubrovskii et al. 1966b pre-dried specimens for 32 hours at a temperature between 110 and $110{ }^{\circ} \mathrm{C}$ followed by hermetically sealing samples before irradiation or thermal exposure. The thermally exposed samples were heated over time to accurately mimic the thermal history of the irradiated samples. In this study, it was found no significant variation in weight loss between the irradiated and thermally exposed samples. Similar results were presented by other authors including Batten 1960 and Rappeneau et al. 1966, among others. Such results indicates no substantial influence of the radiation environment on the long term weight loss of concrete specimens and the weight loss is most likely tied to the loss of free water in the specimens investigated. Studies conducted on neat cement typically reported shrinkage which correlated linearly with the weight loss reported at given fluences, such as the study by Gray (1971)/Kelly et al. (1969), further indicating that weight loss is primarily due to the loss of free water within the specimen.

This is further supported by authors who reported gas generation under irradiation of concrete. The gas generated is mainly composed of hydrogen and oxygen, which is caused by radiolysis and evaporation of the free water in the concrete. Currently, no in situ measurements were made on gas generation in relation to weight loss, making any correlations on radiation accelerating the dehydration of the cement paste difficult. Furthermore, many of the neutron fluxes were low, resulting in specimens being held at high temperature for times longer than required for full dehydration of the paste solely due to the elevated temperature. The limited trends between weight change and fluence within a single study or across multiple studies indicate the weight loss is most likely solely due to dehydration of the cement paste, although the acceleration effect of neutrons or gamma rays could be present but remains weakly explored. Loss of free water could be a contributing factor to the trends shown in Section 3 but does not account for the significant losses of strength observed above a fluence of $1.0 \times 10^{19} \mathrm{n} / \mathrm{cm}^{2}$. 


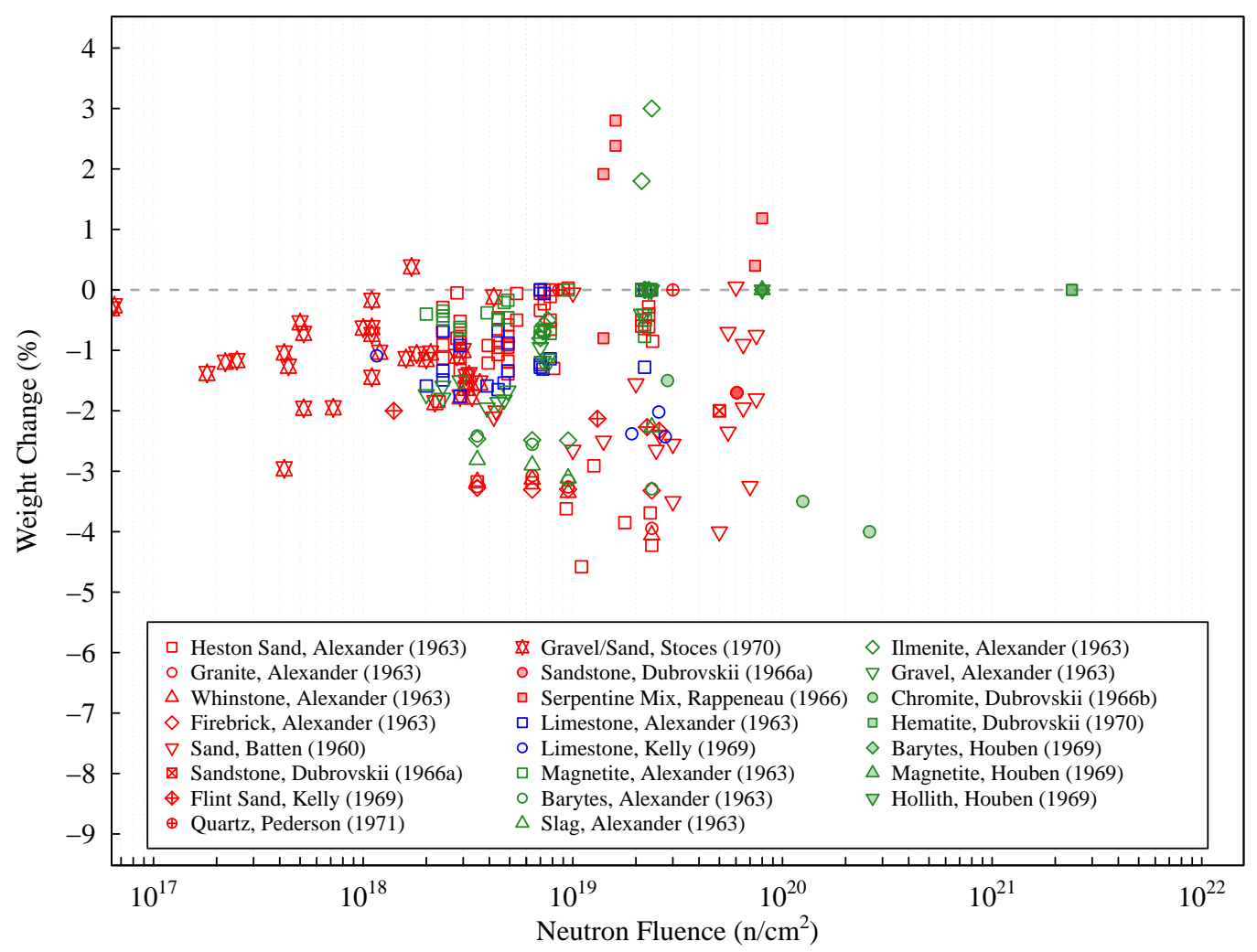

Figure 6: Weight change of concrete and mortar specimens versus neutron fluence. The neutron spectrum and temperature vary between experiments. The neutron spectrum and the specimen temperature vary between experiments. Siliceous concrete is depicted with red symbols, calcareous with blue, and miscellaneous concretes with green. Filled symbols indicate experiments conducted above $100{ }^{\circ} \mathrm{C}$; open symbols indicate experiments conduced below $100{ }^{\circ} \mathrm{C}$. Mix design can be determined by cross referencing with Table 3. Weight loss saturates at $-5 \%$.

\subsection{Irradiated Concrete Dimensional Changes}

As seen in Table 3, many authors reported dimensional variations of irradiated concrete. The change in dimensions of concrete and mortar specimens is a convolution of thermal expansion of the aggregate and cement paste, drying shrinkage, and the effect of neutrons on the aggregate and cement paste. Figure 7 shows the reported volumetric expansion versus neutron fluence with the neutron spectrum, the specimen temperature, and concrete and mortar mixes varying between experiments. Volumetric expansion (swelling) is observed in concretes and mortars at fluences higher than $1.0 \times 10^{18} \mathrm{n} / \mathrm{cm}^{2}$ with marked increases seen at fluences above $1.0 \times 10^{19} \mathrm{n} / \mathrm{cm}^{2}$. It should be noted the expansion observed in Figure 7 for concrete due to irradiation is substantially larger than swelling due to other causes such as an alkali-silica reaction (ASR) or thermal expansion (ISE, 1992).

In general, specimens bearing carbonate and heavy-weight aggregates exhibited smaller volumetric expansions compared to silicate-bearing concretes and mortars at similar neutron fluences and temperature. In particular, Gray (1971)/Kelly et al. (1969), reported limited 
dimensional changes $(\sim 5 \%)$ for concrete with limestone aggregates compared to flint aggregates where at $4.0 \times 10^{19} \mathrm{n} / \mathrm{cm}^{2}$ (fast) flint-aggregate-based concrete showed an increase of $>9 \%$ in volume, while cement paste (ordinary Portland cement) showed a contraction, indicating the paste tends to contract while the concrete as a whole drives towards expansion after high fluence irradiations.

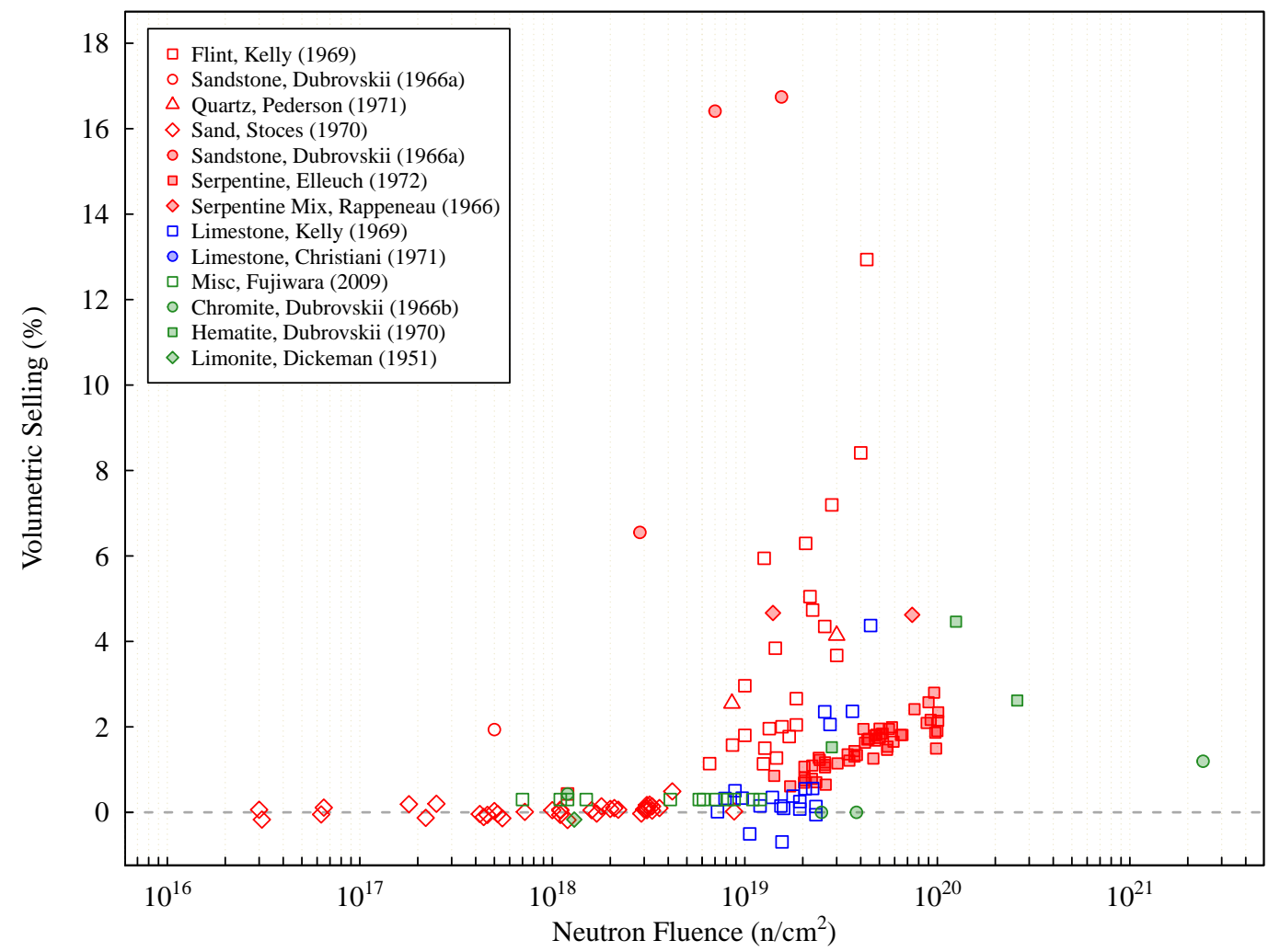

Figure 7: Concrete and mortar volumetric swelling versus neutron fluence. The neutron spectrum and specimen temperature vary between experiments. Filled symbols indicate experiments conducted above 100 ${ }^{\circ} \mathrm{C}$; open symbols indicate experiments conduced below $100{ }^{\circ} \mathrm{C}$.

As mentioned, the expansion is a convolution of several factors including irradiation temperature and neutron-induced material changes. Some irradiated concrete exhibited contraction that can be attributed to shrinkage of the cement paste resulting from the loss of free water or even bonding water at high temperature. Large thermal expansion of the concrete could cause irreversible deformation of the concrete specimen under irradiation. In general, aggregates occupy more than $70 \%$ of the total volume of concrete, resulting in thermal expansion of the concrete being strongly influenced by the aggregate type. For example, Schneider (1981) showed the thermal expansion of siliceous concrete is greater than that for limestone concrete which provides an initially plausible explanation for the differences observed between different concrete types. Given this, and assuming thermal expansion results in irreversible deformation, the expansions observed for both siliceous 
and calcareous concrete above $1 \times 10^{19} \mathrm{n} / \mathrm{cm}^{2}$ exceed typical irreversible expansions observed based on commonly reported thermal expansions for concrete of any mix design (U.S. Bureau of Reclamation, 1988), indicating an influence of neutron irradiation on the expansion of irradiated concrete.

In the case of aggregates, several studies published dimensional changes of aggregates as well as dimensional changes of concrete or mortars. Figure 8 shows the expansion of the aggregates (not embedded in concrete) from these studies. Figure 8 indicates swelling of aggregates can be observed in the complex minerals irradiated up to $3.0 \times 10^{20} \mathrm{n} / \mathrm{cm}^{2}$. In general, siliceous aggregates demonstrated larger swelling than calcareous or heavy weight aggregates, a finding observed by both Gray (1971)/Kelly et al. (1969) and Seeberger and Hilsdorf (1982). Comparison of Figure 7 with Figure 8 shows a potential correlation between the swelling of the irradiated aggregates for a given fluence and the observed swelling seen in irradiated concrete and mortar specimens. This correlation suggests the expansion of aggregates is likely an indicator of the expansion of concrete and mortars shown in Figure 7. The limited swelling of aggregates below $1.0 \times 10^{19} \mathrm{n} / \mathrm{cm}^{2}$ (for all temperatures) indicates the effect is likely due to neutron radiation. The mechanisms for such expansion and relationship to degradation of irradiated concrete structures are discussed in Section 6.

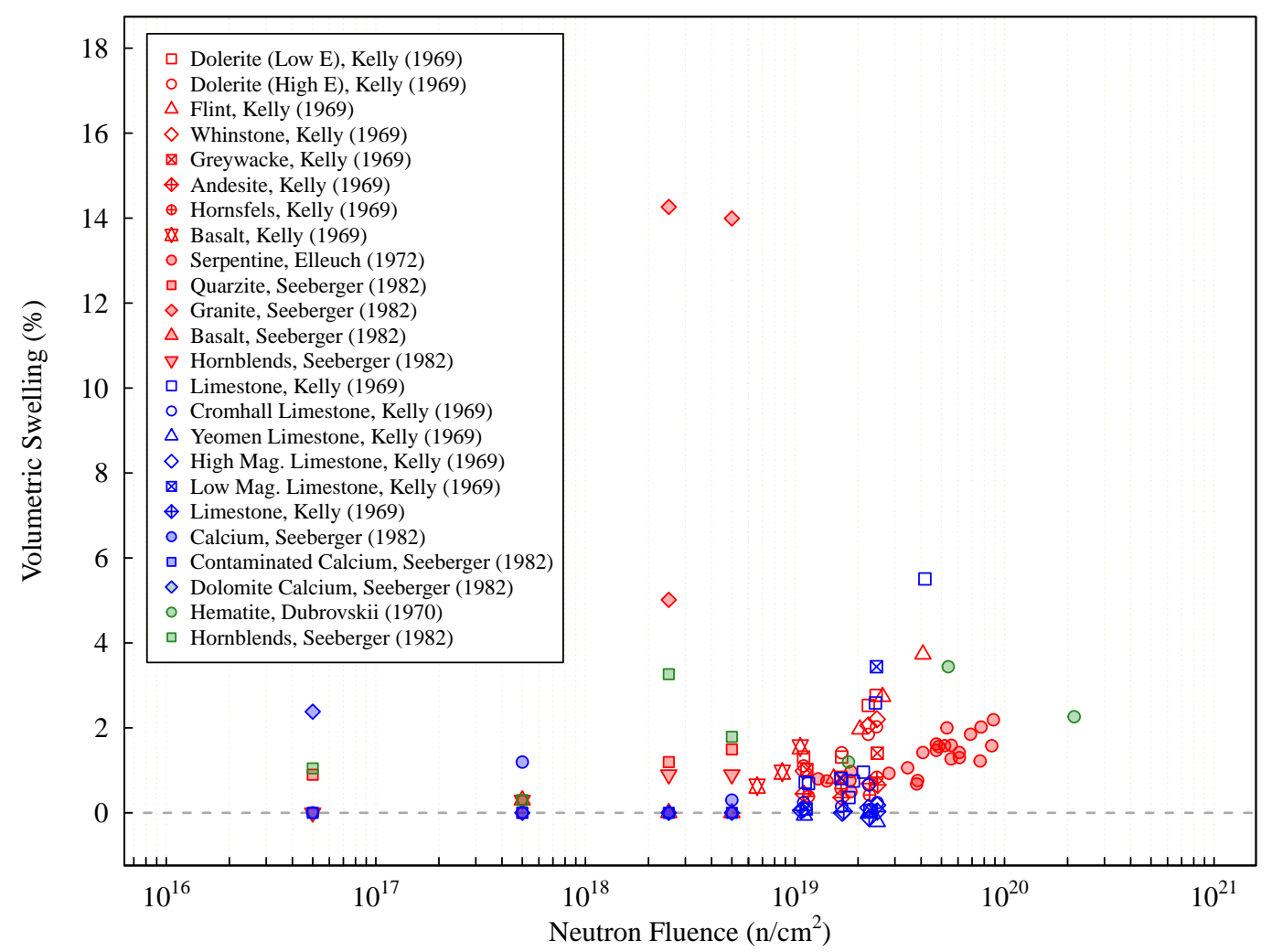

Figure 8: Aggregate volumetric swelling versus neutron fluence. The neutron spectrum and specimen temperature vary between experiments. Filled symbols indicate experiments conducted above $100{ }^{\circ} \mathrm{C}$; open symbols indicate experiments conduced below $100{ }^{\circ} \mathrm{C}$. 


\section{Limitations in Presented Data}

Some limitations within the data should be recognized. The majority of the mechanical property data for neutron fluences greater than $1.0 \times 10^{19} \mathrm{n} / \mathrm{cm}^{2}$ were obtained at temperatures $>100{ }^{\circ} \mathrm{C}$ (filled symbols), limiting their practical use for LWR interpretations at face value since the temperature in biological shield structures or RPV support structures is bounded by design at $65{ }^{\circ} \mathrm{C}$ with peak values not exceeding $93{ }^{\circ} \mathrm{C}$. As discussed within the previous sections, elevated temperature in the absence of radiation can also have detrimental impacts on the mechanical behavior of concrete and mortars. Therefore, data above $>100{ }^{\circ} \mathrm{C}$ is a superposition of both the deleterious effects of neutron irradiation and time at elevated temperature.

Another limitation is the presented data are not normalized to a specific neutron energy cut-off, such as E $>0.1 \mathrm{MeV}$, resulting in the possibility of data points having higher or lower fluence levels than those reported if they were indeed normalized consistently. This is highlighted in Figure 9 where Figure 2 has been re-colored based on the reported neutron energy cut-off reported in Table 3. Of particular importance are the data highlighted in yellow, which represent data where $\mathrm{E}>1.0 \mathrm{MeV}$ and the driving force for the observed "knee" in the data at $1 \times 10^{19} \mathrm{n} / \mathrm{cm}^{2}$. Unfortunately, these data points do not coincide with a reported neutron flux spectrum, and therefore, the ratio of fast neutrons to thermal neutrons remains unknown. Given this, normalization to either $\mathrm{E}>0.1 \mathrm{MeV}$ or the integral neutron fluence would result in an increase in the reported neutron fluence for each data point and shift the yellow data points towards higher neutron fluences in Figure 9. Similar assessments can be made on data points reported using an integrated neutron fluence (green diamonds in Figure 9). This sub-set of data would shift to lower neutron fluence if the data were normalized to either $\mathrm{E}>0.1 \mathrm{MeV}$ or $\mathrm{E}>1.0 \mathrm{MeV}$. These observations show the reported energy cutoff is crucial for the assessment of concrete degradation and the need for an accepted integrated damage parameter, such as displacements per atom (dpa), to fully assess the effects of radiation on concrete and mortar specimens. Unfortunately, as seen in Table 3, only a few authors published the neutron flux spectrum for the test positions utilized within their studies. Due to insufficient information in the literature, any attempt to normalize the data or determine a viable integrated damage parameter based on empirical evidence at this time remains futile. 


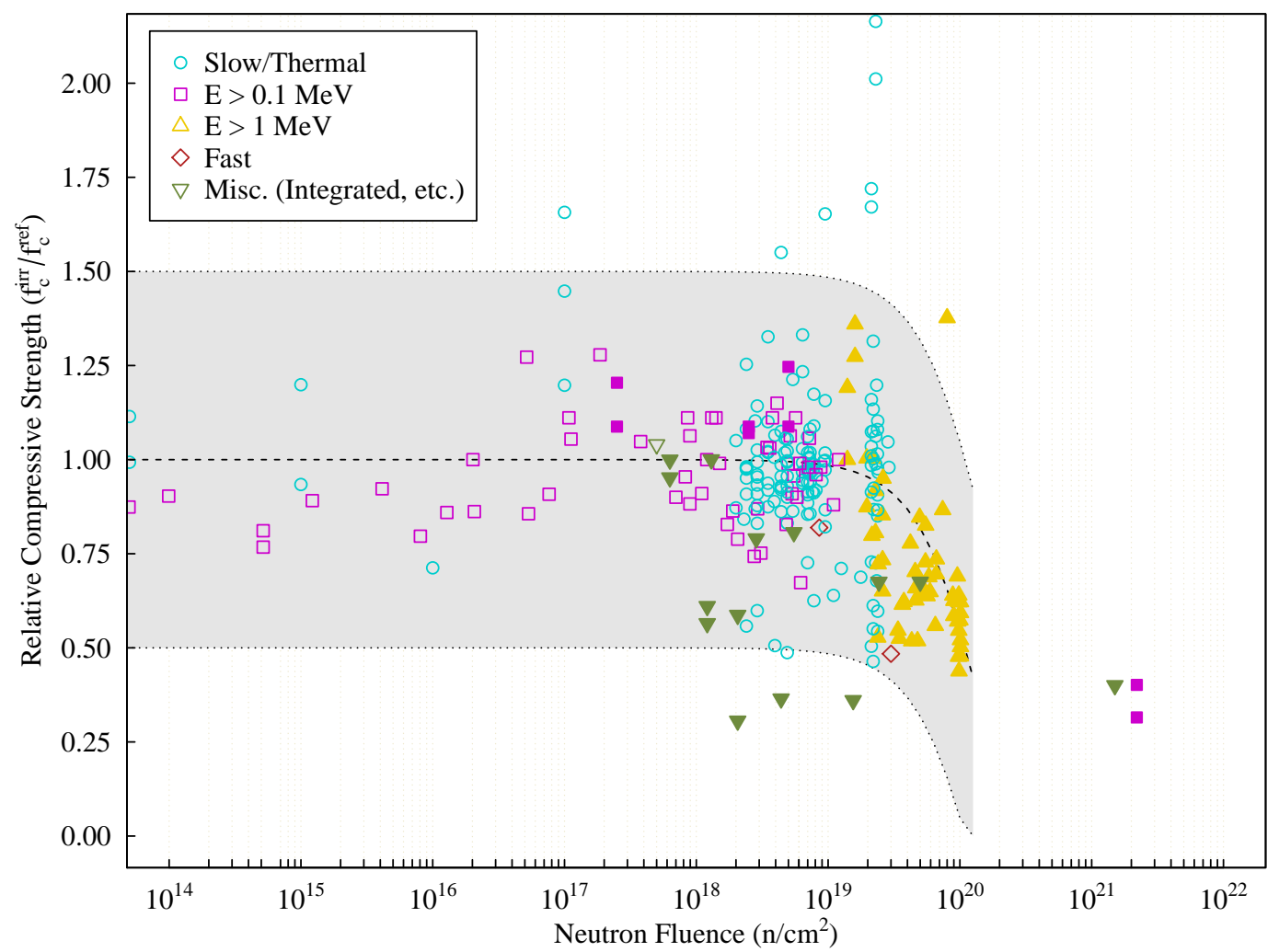

Figure 9: Relative compressive strength of concrete and mortar specimens versus neutron fluence. The neutron spectrum and specimen temperature vary between experiments. Filled symbols indicate experiments conducted above $100{ }^{\circ} \mathrm{C}$, open symbols indicate experiments conduced below $100{ }^{\circ} \mathrm{C}$. Coloring scheme adapted to depict reported neutron energy reference level.

The relative mechanical properties data for irradiated specimens are given in terms of the values for non-irradiated reference specimens. This introduces bias between different studies and increases the variance in the data presented in Figures 2-8 as the condition of reference samples used to determine mechanical and physical properties for unirradiated specimens varied greatly between authors. For instance, the values presented by Idei et al. (1990) are based on a 28 day cure of the concrete, while the data from Alexander (1963) are based on samples not aged at elevated temperatures, while data of Seeberger and Hilsdorf (1982) are. Also, the data include different specimen geometries with dimensions often lower than the standards' recommended sizes. Smaller sized samples can increase the variance of the experimental results (Neville, 1963) and contribute to the large scatter bands within the data presented in Figure 2-8. This scatter band is also exacerbated due to the inherent variability of the material properties (fabrication) and testing equipment from one laboratory to the other.

Another source of the large scatter in the data presented is the formulation of the concrete. For example, variations are observed in the aggregate-to-cement ratio from author to author, as well as the water-to-cement ratio. These variances in mix design, as mentioned for tensile strength, can significantly impact the mechanical and physical property 
results and could vary the irradiation response as well. Finally, it should also be noted that the radiation test might produce gradients of temperature and fluence through the sample which were not addressed by the original authors and could not be considered here. All these factors serve to increase the experimental scatter, although the increased amount of presented data over previously published trends helps to increase confidence in conclusions previously suggested in the literature.

\section{Mechanism for Radiation-Induced Volumetric Expansion (RIVE) in Concrete Structures}

\subsection{Radiation Damage Processes in Non-Metals}

The results shown in Section 4.2 indicate the dimensional changes of irradiated concrete are correlated with the irreversible volumetric swelling of aggregates embedded in concrete. This effect, as noted in the companion paper, is a convolution of thermal expansion and radiation-induced swelling of the aggregate during accelerated irradiation testing. For the sake of describing the overall swelling mechanism for concrete where the expansion and/or contraction of both the paste and aggregate must be considered and distinguishing it from chemically induced expansions such as ASR, the term, "radiation-induced volumetric expansion" (RIVE) is coined. Of primary importance to concrete for LWRs is radiation-induced effects as the temperature of the biological shield is maintained at relatively low temperatures $\left(<100{ }^{\circ} \mathrm{C}\right)$ under operation.

Concrete aggregates are typically well-crystalized non-metallic mineral structures due to the nature of their sourcing. The radiation effects in non-metals is inherently complex, with numerous reviews being published on the matter (e.g., Clinard and Hobbs, 1986; Hobbs et al., 1994; Zinkle and Kinoshite, 1997; Weber et al., 1998; Was, 1997; Konings, 2012). To summarize, the effect of radiation, in relation to concrete structures, can be subdivided into three important topics: (1) the primary damage event, (2) secondary defect formation, and (3) structural effects. The primary damage event results in displaced atoms which form Frenkel (vacancy-interstitial) pairs and can be produced either by direct momentum transfer (ballistically) or by electronic excitation (radiolytically). The displacement response will vary from one phase to another and even within a phase due to each atom type having a different displacement probability (Zinkle and Kinoshite, 1997). The response to the primary damage event can include defect recombination, defect aggregation, and amorphization, and these responses can be strongly affected by electrostatic and covalent interactions.

The formation of discrete defects in a crystalline lattice can cause structural effects including swelling. Non-metals have been shown to exhibit dilatational swelling up to several percent, depending on the material and irradiation conditions. Swelling can also occur due to amorphization as the densely packed crystalline state converts to a less-dense state with limited long-range order. This phenomena has been well documented for different material systems and can exceed the swelling caused due to discrete defects. Both effects will most likely be exacerbated due to the low-temperature irradiations observed in biological shields, which will limit defect recombination. Limited literature exists to fully evaluate the radiation damage process and response of those aggregates used in Table 4, but initial analysis shows the expansions observed in Section 4.2 is due to radiation damage of the aggregates. This process can be best explained using mineral analogues, such as $\alpha$-quartz and calcite, to describe the mechanisms for concrete degradation in the presence of neutron irradiation and the resulting effects on mechanical properties. The following section summarizes such analysis. 


\subsection{Swelling due to Amorphization in Simple Mineral Analogues}

Silicates are a common aggregate type due to their naturally occurring abundance. The simplest form of a silicate aggregate is pure, single-crystal $\alpha$-quartz. Radiation effects of single-crystal $\alpha$-quartz have been reported under ion and fast-neutron irradiation with numerous review articles published on the matter (e.g., Bonnet et al., 1994; Douillard and Duraud, 1996; Hobbs et al., 1994; Wang et al., 2000). To summarize, the global response of $\alpha$-quartz to neutron induced radiation damage, and many $\mathrm{SiO}_{4}$ tetrahedra crystalline systems, is the amorphization due to the loss of long-range order. As summarized by Douillard and Duraud (1996), at low neutron fluences $\left(\leq 1.0 \times 10^{18} \mathrm{n} / \mathrm{cm}^{2}\right)$, the neutrons generate point defects with the concentration increasing as a power function to fluence. Until neutron fluences $\geq 6.0 \times 10^{19} \mathrm{n} / \mathrm{cm}^{2}, \alpha$-quartz undergoes a phase transition from $\alpha$-quartz to $\beta$-quartz. This transition is reversible, with the initial state recovered via annealing. At fluences above $6.0 \times 10^{19} \mathrm{n} / \mathrm{cm}^{2}$, the $\alpha$-quartz phase is lost and the remaining structure is a composite of $\beta$-quartz and amorphous quartz.

The amorphization process of irradiated single crystal $\alpha$-quartz has been shown to be directly related to a density decrease with a maximum at $\sim 14 \%$ when complete amorphization is achieved (Primack, 1958). The decrease in density is directly related to volumetric swelling, and the experimental data shows a sigmoidal response. The volumetric swelling due to amorphization is irreversible and maintained after cooling of the specimen. Bolse (1998) found that the evolution of the fraction $(\chi)$ of disordered atoms in $\alpha$-quartz after irradiation with $50 \mathrm{keV} \mathrm{Na}{ }^{+}$ions at $77 \mathrm{~K}$ was correctly reproduced by a nucleation-growth type model (Avrami, 1941):

$$
\chi(n)=1-\exp \left\{-(K n)^{d}\right\},
$$

where $n$ is the ion fluence, $K$ and $d$ are a temperature-dependent parameter and a dimensionality factor, respectively, where $d$ can vary between 2 and 5 . Other sigmoidal models may also be used (Zubov and Ivanov, 1966) (see companion article), but the nucleation and growth model offers a simple approach towards a mechanistic understanding. The nucleation and growth model can also capture swelling in neutron irradiated $\alpha$-quartz. Figure 10 plots the volumetric expansion and fraction of disordered atoms of $\alpha$-quartz from the literature at various neutron fluence, energy, and temperature. Swelling of crystalline $\alpha$-quartz starts at $\sim 1.0 \times 10^{19} \mathrm{n} / \mathrm{cm}^{2}(\mathrm{E}>0.1 \mathrm{MeV})$ with full amorphization and swelling ( 14-15 vol.\%) observed at $\sim 2.0 \times 10^{20} \mathrm{n} / \mathrm{cm}^{2}(\mathrm{E}>0.1 \mathrm{MeV})$. The range of fluences where swelling in $\alpha$-quartz occurs coincides with ranges observed in Figure 8, where significant swelling in most siliceous aggregates was observed to start at $\sim 1.0 \times 10^{19} \mathrm{n} / \mathrm{cm}^{2}$, indicating a possible correlation between the amorphization of silicate phases and swelling seen in aggregates. The experimental data presented for swelling in $\alpha$-quartz includes irradiation temperatures between $38{ }^{\circ} \mathrm{C}$ to $300{ }^{\circ} \mathrm{C}$ and the good agreement across these test temperatures indicates

at the temperatures relevant to LWRs the displaced atoms do not have sufficient mobility to reorder to maintain crystallinity and will result in swelling of siliceous aggregates. 


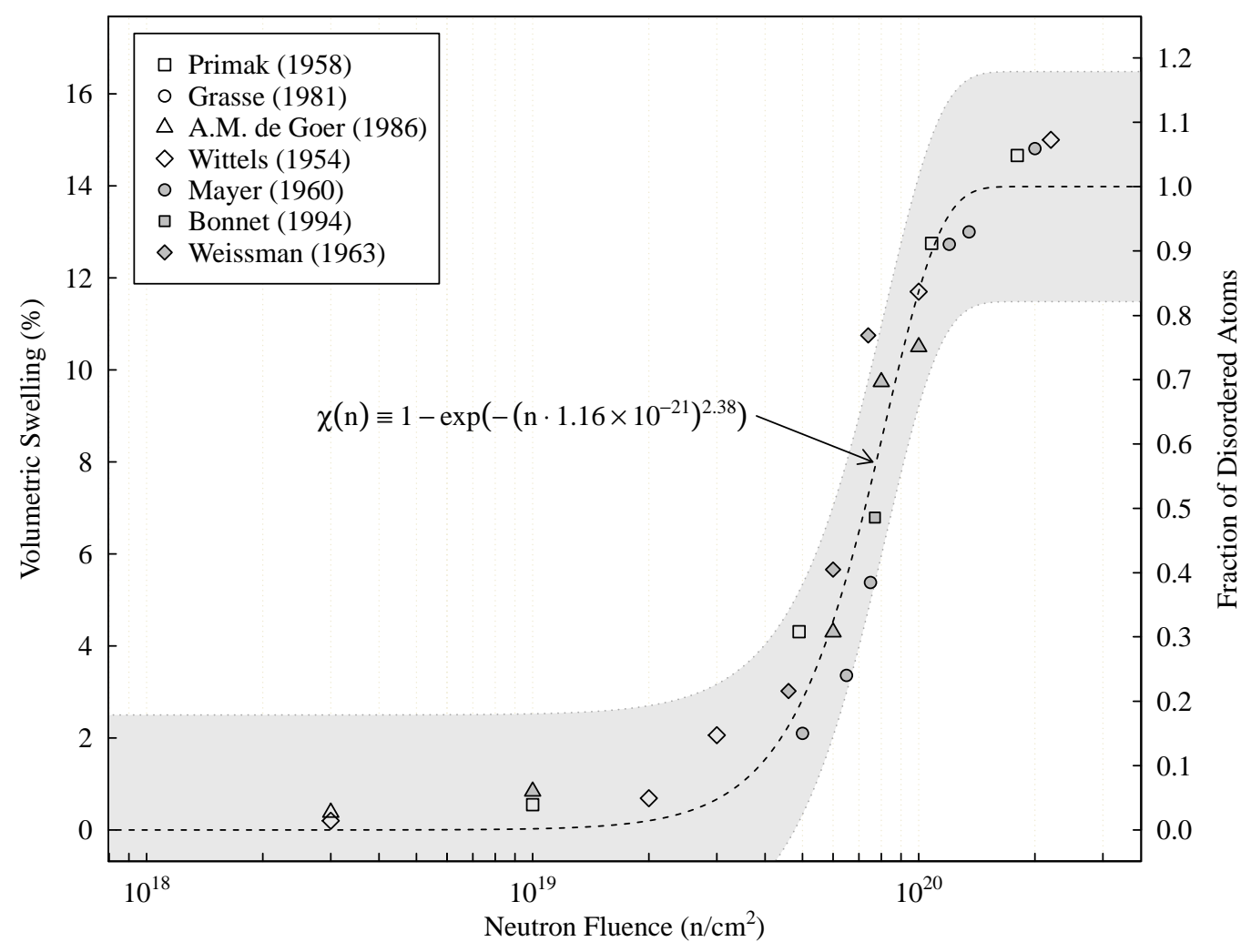

Figure 10: Volumetric swelling and fraction of disordered atoms of $\alpha$-quartz versus neutron fluence. The neutron spectrum and specimen temperature vary between experiments. Filled symbols indicate experiments conducted above $100{ }^{\circ} \mathrm{C}$; open symbols indicate experiments conduced below $100{ }^{\circ} \mathrm{C}$. Trend line determined using non-linear square fit to Eq. 2; shaded region indicates $95 \%$ prediction interval to the fitted function.

The swelling observed in aggregates shown in Figure 8 showed a quite complex response with the magnitude and initiation fluence varying between aggregates. This response is most likely due to variances in the radiation response of different phases present in siliceous aggregates. More complex silicates have been studied using energetic ions and show large variances in the radiation response with several important variables intrinsic to the silicate type driving the variations in radiation response. For example, Eby et al. (1992) conducted a comprehensive series of ion-beam irradiation tests on silicate minerals and found that the critical amorphization dose (fluence) is best correlated with the melting point (a physical parameter related to bonding, structure, and composition of the crystalline phase), a structural property such as the atomic packing or the dimensionality of silica polymerization describing the crystalline structure (i.e., isolated, chain, sheet, 3D interconnected framework silicate), and a bonding property like ionicity. Of the different types of oxygen bonds in silicate structures, the Si-O bond is the weakest. Seeberger and Hilsdorf (1982) suggested that muscovite, a form of mica (i.e., a layered silicate) exhibiting large lattice constant changes under irradiation, could be the cause of the severe degradation observed on two tested granites at a fluence level between $\sim 1.0 \times 10^{19} \mathrm{n} / \mathrm{cm}^{2}$ (fast) and $\sim 5.0 \times 10^{19} \mathrm{n} / \mathrm{cm}^{2}$ (fast) seen in Figure 8. Templier et al. (1996) also observed the formation of large voids in mica that 
could possibly explain high expansions perpendicular to the basal plane of phlogopite micas in the absence of radiation-induced amorphization.

Expanding towards other mineral classes, including carbonates, further shows the complexity in developing an understanding of the radiation effects on the microstructure of irradiated concrete. For example, the simplest form of carbonates is calcite. Experimental neutron damage data on calcite are sparser than $\alpha$-quartz and complex silicates. Wong (1974) indicated a density decrease of $\sim 0.3 \%$ at a total integrated neutron fluence of 1.0x $10^{20}$ $\mathrm{n} / \mathrm{cm}^{2}$, while other authors (Luthra, 1969; Hauser and Schenik, 1964) have reported no change in lattice constants or density at fluences up to $2.8 \times 10^{19} \mathrm{n} / \mathrm{cm}^{2}(\mathrm{E}>0.1 \mathrm{MeV})$. Wong (1974) speculated the radiation tolerance of pure calcite is due to the ionic bonding nature of the crystal compared to the strong covalent nature of $\alpha$-quartz, a finding similar to those of Seeberger and Hilsdorf (1982) or Eby et al. (1992). The limited swelling observed for carbonates in Figure 8 compared to silicates at the same neutron fluence could be due to the superior radiation tolerance of calcite as reported in literature.

The variation in the radiation response of simple single-crystal systems, which can serve as analogues for more complex mineralogical specimens, indicates the overall complexity of the radiation damage process. It also highlights the potential for the degree of amorphization and hence volumetric expansion to vary depending on the aggregate type and even on the volume fraction of phases present in the aggregates used. This is particularly problematic when comparing single subsections of aggregates, such as limestone, where the minor amounts of quartz or feldspar could significantly impact the matrix primarily composed of calcite. As such, the simple partitioning scheme used within this paper, to a degree, could be an over simplification but offers at least an initial scheme to partition the effects of radiation for different concrete designs and begin understanding first-order mechanism(s) for degradation of irradiated concrete.

\subsection{Influence of Radiation-Induced Volumetric Expansion on Mechanical Properties}

At a given neutron fluence, concrete expansion is of the same order of amplitude as that of the aggregate it is made of, as seen in Figures 7 and 8. The relationship between the aggregate expansion, cement paste contraction, and corresponding RIVE of concrete is detailed in the companion paper (Le Pape et al., 2014). Only an overview is provided here for completeness. The decrease in the mechanical properties at neutron fluences higher than $\sim 1.0 \times 10^{19} \mathrm{n} / \mathrm{cm}^{2}$ coincides with the development of $\alpha$-quartz amorphization suggesting a possible interaction between neutron-induced amorphization of aggregate, RIVE, and the loss of concrete mechanical properties. It is estimated that the fast-neutron fluences in the biological shields of numerous LWRs will exceed $1.0 \times 10^{19} \mathrm{n} / \mathrm{cm}^{2}$ during nominal plant design lifetime and/or extended operation based on the analysis in Section 2.2. Therefore, the effects of irradiation on concrete need to be considered in evaluating the long-term operation of LWR power plants.

Based on the analysis above, and the model used for deconvolution of competing effects presented in the companion paper, an interpretation of the influence of RIVE in absence of elevated temperatures on the mechanical properties of irradiated concrete can be suggested: (i) neutron irradiation has no or limited first order effects on the cement paste in the range of $1.0 \times 10^{14}-10^{22} \mathrm{n} / \mathrm{cm}^{2}$ (Kontani et al., 2010), (ii) neutron irradiation leads to amorphization of crystalline phases, with certain phases being more susceptible to amorphization than others (Eby et al., 1992; Seeberger and Hilsdorf, 1982), (iii) the degree of RIVE due to amorphization is material dependent (Gray, 1971; Kelly et al., 1969; Hilsdorf 
et al., 1978; Seeberger and Hilsdorf, 1982) (iv) significant RIVE of concrete will impart irreversible damage, resulting in a marked decrease in mechanical properties.

The damage imparted into the concrete can be characterized as micro-cracking in the cement paste due to differential strains between the cement paste and aggregate or microcracking in the aggregate due to anisotropic swelling in the aggregate due to different swelling responses of the phases which are present in the aggregate of interest. Micro-cracking in the paste due to differential swelling was observed and reported by numerous authors (Seeberger and Hilsdorf, 1982; Kelly et al., 1969; Gray, 1971; Dubrovskii et al., 1968, 1966a), while micro-cracking in the aggregate has also been reported (Seeberger and Hilsdorf, 1982) and observed in other non-metal systems such as $\mathrm{BeO}$ (Hickman, 1968). Micro-cracking in the aggregate could explain the significant loss of tensile strength seen in carbonate aggregates with limited swelling reported by Gray (1971)/Kelly et al. (1969). The exact amount of micro-cracking in the paste and/or aggregate at a specific neutron fluence remains ambiguous as it is hypothesized that many factors contribute to the magnitude of RIVE at a specific neutron fluence including phases present, mix design, irradiation temperature, neutron flux (damage dose rate), sample confinement, among others. Nevertheless, the swelling of aggregates appears to be a probable first-order mechanism for degradation of irradiated concrete structures, although the correlations between concrete degradation and neutron irradiation have been demonstrated to be quite complex, with areas still ripe for investigation to verify the mechanism described herein.

\section{Conclusions}

The current state of the art of neutron-irradiated concrete was reviewed from the perspective of long-term operation of concrete structures in typical LWR power plants. Based on the analysis above, several important conclusions can be made.

- Concrete irradiated with neutrons can see a marked decrease in compressive strength at fluence levels above $1.0 \times 10^{19} \mathrm{n} / \mathrm{cm}^{2}$ with lower bounds at $50 \%$ of the reference concrete strength.

- Neutron irradiation of concrete has a greater impact on the tensile strength compared to the compressive strength. Loss of tensile strength occurs at fluence levels similar to those for compressive strength, but a $75 \%$ loss of tensile strength can occur compared to a $50 \%$ loss of compressive strength. As a result, the usual relationships (code or empirical formulae) used to derive the tensile strength in absence of measured compressive strength may not be applicable.

- The elastic modulus shows a gradual decrease under neutron irradiation with significant decreases observed above $1.0 \times 10^{19} \mathrm{n} / \mathrm{cm}^{2}$, although data reported in this range were also conducted at high temperature, and the combined effect of neutrons and elevated temperature must be considered.

- The decrease in mechanical properties is attributed to many factors but indications show that the RIVE is a first-order mechanism for loss of mechanical properties under neutron irradiation. Radiation tolerance is expected to be dependent on the aggregate type and a partitioning scheme for evaluating the data was presented. Data suggest siliceous aggregates present the highest risk for deleterious effects of both irradiation and elevated temperatures on concrete due to its susceptibility for low fluence amorphization and corresponding swelling and high thermal expansion coefficients. 
- The estimated fast neutron fluences $(\mathrm{E}>0.1 \mathrm{MeV})$ in the biological shield during longterm operation (>40 years) will reach the levels where the effect of RIVE and loss of mechanical properties are expected and cannot be ignored as a potential degradation mechanism for LWR biological shields. A risk assessment would require knowledge of the concrete mix design (w/c ratio, aggregate type, operating temperature, etc.), the radiation tolerance and response of the aggregate, and the neutron environment including the neutron spectrum and fluence profile through the biological shield.

The existing irradiated concrete database appears to clearly indicate the appearance of RIVE and compressive and tension strength degradation of concrete under neutron irradiation. However, the effects of the important parameters, such as neutron fluence magnitude at which RIVE becomes significant, the neutron fluence energy cutoff associated with said fluence level, the effect of elevated concrete temperature during irradiation, and many variables associated with the concrete mix and aggregates, cannot be readily separated out and clearly evaluated from the existing data. Further research, carefully designed to target selected parameters, is necessary to fill the knowledge gaps. This review serves as a next step towards a fundamental understanding of the key variables associated with radiationinduced degradation in concrete structures, and potentially a catalyst for further research on the topic.

\section{Acknowledgments}

The authors would like to thank Dr. D. Naus, Dr. T.M. Rosseel and Dr. J.T. Busby for their thoughtful discussions on the presented topics. This research is sponsored by the Department of Energy Light Water Reactor Sustainability Program. This manuscript has been authored by the Oak Ridge National Laboratory, managed by UT-Battelle LLC under Contract No. DE-AC05-00OR22725 with the U.S. Department of Energy. The publisher, by accepting the article for publication, acknowledges that the U.S. Government retains a nonexclusive, paid-up, irrevocable, worldwide license to publish or reproduce the published form of this manuscript, or allow others to do so, for U.S. Government purposes.

\section{References}

ACI Committee 349, 2002. Evaluation of Existing Nuclear Safety-Related Concrete Structures. Technical Report ACI 349.3R-02. American Concrete Institute.

Alexander, S., 1963. Effects of Irradiation on Concrete Final Results. Technical Report HL.63/6438. Atomic Energy Research Establishment. Harwell.

Avrami, M., 1941. Granulation, phase change, and microstructure kinetics of phase change. iii granulation, phase change, and microstructure kinetics of phase change. Journal of Chemical Physics 9, 177-184.

Batten, A., 1960. Effect of Irradiation on the Strength of Concrete. Technical Report AERE-R 3332. Atomic Energy Research Establishment. Harwell.

Blosser, T., Bond, G., Lee, L., Morgan, D., Nichols, J., Reid, R., Reynolds, A., Speidel, T., Vroom, D., Welt, M., 1958. A Study of the Nuclear and Physical Properties of the ORNL Graphite Reactor Shield. Technical Report ORNL-2195. Oak Ridge National Laboratory. Oak Ridge, TN. 
Bolse, W., 1998. Formation and development of disordered networks in si-based ceramics under ion bombardment. Nuclear Instruments and Methods in Physics Research Section B: Beam Interactions with Materials and Atoms 141, 133-139.

Bonnet, J., Boissier, M., Gherbi, A.A., 1994. The amorphization process of neutronirradiated crystalline quartz studied by brillouin scattering. Journal of Non-Crystalline Solids 167, 199-204.

British Standards Institute 4975, 1990. Specification for Prestressed Concrete Pressure Vessels for Nuclear Reactors. Technical Report BS 4975:1990. "British Standards Institute".

Christiani, S., Granata, S., Montagnini, A., 1971. Behaviour of plastic mortar samples under temperature and radiation conditions, in: Benzler, H. (Ed.), Proceedings of an Information Exchange Meeting on 'Results of Concrete Irradiation Programmes', Commission des Communautés Européennes, Brussels, Belgium.

Clark, R., 1958. Radaiation Damage to Concrete. Technical Report HW-56195. General Electric. Hanford Laboratories, Richland WA.

Clinard, F., Hobbs, L., 1986. Modern Problems in Condensed Matter Sciences. NorthHolland Physics Publishing. volume Physics of Radiation Effects in Crystals. chapter Radiation Effects in Non-Metals.

Crispino, E., Fizzotti, C., Gasparini, R., Qualtieri, G., Montagnini, A., Rossi, F., 1971. Irradiation effects on reactor structural materials, in: Fourth United Nations International Conference on the Peaceful Uses of Ataomic Energy, p. 22.

Dickeman, R., 1951. Technical Activities Report - Pile Physics. Technical Report HW-22132.

Diererichs, C., Ehm, C., 1981. Effect of temperature on steel and concrete for pcrv's. Nuclear Engineering and Design 67, 245-258.

Douillard, L., Duraud, J., 1996. Amorphization of a-quartz under irradiation. Journal De Physique III , 1677-1687.

Dubois, F., Mauny, P., Bernard, A., Elleuch, M., 1969. Nouveaux types de betons calorifuges pour reacteurs nucleaires, in: Proceedings of the Second Information Meeting on Pre Stress Concrete and Reactor Pressure Vessels and their Thermal Isolation, Brussels, Belgium. pp. 975-1018.

Dubrovskii, V., Ibragimov, S., Korenevskii, V., Ladygin, A., Pergamenshchik, B., Perevalov, V., 1970. Hematite concrete for shielding against high neutron fluxes. Atomnaya Energiya $28,258-260$.

Dubrovskii, V., Ibragimov, S., Kulakovskii, M.Y., Pergamenshchik, A.Y.L.B., 1966a. Radiation damage in ordinary concrete. Atomnaya Energiya 23, 310-316. English Translation.

Dubrovskii, V., Ibragimov, S., Ladgyn, A.Y., Pergamenshchik, B.K., 1966b. The effect of neutron irradiation on certain properties of refractory concretes. Atomnaya Energiya 21, 108-112. English Translation.

Dubrovskii, V., Ibragimov, S., Ladygin, A., Kulakovskii, M., Pergamenshchik, B., 1968. Radiation stability of serpentine concrete. Soviet Atomic Energy 25, 1345-1346. 
Eby, R., Ewing, R., Birtcher, R., 1992. The amorphization of complex silicates by ion-beam irradiation. Journal of Materials Research 7, 3080-3102.

Elleuch, L., Dubois, F., Rappenau, J., 1971. Behavior of special shielding concretes and of their constituents under neutron irradiation, in: Fourth United Nations International Conference on the Peaceful Uses of Ataomic Energy, p. 7.

Elleuch, L., Dubois, F., Rappeneau, J., 1972. Effects of neutron radiation on special concretes and their components. Special Publication of The American Concrete Institute 43, 1071-1108.

Evans, T., Stafford, A., Slaybaugh, R., Clarno, K., 2010. Denovo: A new three-dimensional parallel discrete ordinates code in scale. Nuclear Technology 171, 171-200.

Feret, R., 1892. Sur la compacité des mélanges hydrauliques. Annales des Ponts et Chaussées $4,5-164$.

Fillmore, D., 2004. Literature Review of the Effects of Radiation and Temperature on the Aging of Concrete. Technical Report INEEL/EXT-04-02319. Idaho National Engineering and Environmental Laboratory.

Freskakis, G., 1980. Behavior of reinforced concrete at elevated temperatures, in: ASCE Conference on Civil Engineering and Nuclear Power, American Society of Civil Engineers, Knoxville, TN. pp. 1-21.

Fujiwara, K., Ito, M., Sasanuma, M., Tanaka, H., Hirotani, K., Onizawa, K., Suzuki, M., Amezawaf, H., 2009. Experimental study of the effect of radiation exposure to concrete, in: Proceedings of the 20th International Conference on Structural Mechanics in Reactor Technology, Espoo, Finland.

de Goer, A., 1986. Phonon scattering in disordered systems, in: Phonon Scattering in Condensed Matter V, pp. 6-14.

Granata, S., Montagnini, A., 1971. Studies on behavior of concretes under irradiation, in: Kesler, C. (Ed.), Concrete for Nuclear Reactors. American Concrete Institute. volume 34, pp. 1163-1172.

Grasse, D., Kocar, O., Peisi, H., Moss, S., Golding, B., 1981. Diffuse x-ray scattering and phonon echoes from neutron irradiated crystalline quartz. Physical Review Letters 46, 261-264.

Graves, H., Le Pape, Y., Naus, D., Rashid, J., Saouma, V., Sheikh, A., Wall, J., 2013. Expanded Material Degradation Assessment (EMDA), Volume 4: Aging of Concrete. Technical Report NUREG/CR-7153, ORNL/TM-2011/545. U.S. Nuclear Regulatory Commission.

Gray, B., 1971. The effects of reactor radiation on ccement and concrete, in: Benzler, H. (Ed.), Proceedings of an Information Exchange Meeting on 'Results of Concrete Irradiation Programmes', Commission des Communautés Européennes, Brussels, Belgium.

Halliday, D., 1956. Heat Release in Concrete Reactor Shields. Technical Report SWP/P 31. Atomic Energy Research Establishment. Harwell. 
Hauser, O., Schenik, M., 1964. Veränderungen der kristallstruktur einiger oxyde, karbonate und titanate durch neutronenbestrahlung. Physica Status Solidi (b) 6, 83-88.

Hickman, B., 1968. Radiation effects in beryllium and beryllium oxide. Studies in Radiation Effects .

Hilsdorf, H., Kropp, J., Koch, H., 1978. The effects of nuclear radiation on the mechanical properties of concrete. Special Publication of The American Concrete Institute 55, 223254.

Hobbs, L., F.W. Clinard, J., Zinkle, S., Ewing, R., 1994. Radiation effects in ceramics. Journal of Nuclear Materials 216, 291-321.

Hookham, C., 1995. In-Service Inspection Guidelines for Concrete Structures in Nuclear Power Plants. Technical Report ORNL/NRC/LTR-95/14. Oak Ridge National Laboratory.

Houben, J., 1969. I. de bestraling van mortelproefstukken, in: Second information meeting on prestressed concrete reactor pressure vessels and their thermal isolation, Commissions of the European Communities.

Idei, Y., Kamata, H., Akutsu, Y., Onizawa, M., Nakajima, N., Sukegawa, T., Kakizaki, M., 1990. Strength of biological shield concrete of JPDR. Technical Report JAERI-M 90-205. Japan Atomic Energy Research Institute.

ISE, 1992. Structural Effects of Alkali-Silica Reaction - Structural Effects of Alkali-Silica Reaction - Technical Guidance Appraisal of Existing Structures. Technical Report. Institution of Structural Engineers (ISE). London, United Kingdom.

Janotka, I., Nürnbergerová, T., 2005. Effect of temperature on structural quality of the cement paste and high-strength concrete with silica fume. Nuclear Engineering and Design 235, 2019-2032.

Kaplan, M., 1983. Nuclear Radiation and the Properties of Concrete. Technical Report UCT-NLSMRU-TR-35. University of Cape Town.

Kelly, B., Brocklehurst, J., Mottershead, D., McNearney, S., 1969. The effects of reactor radiation on concrete, in: Proceedings of the Second Information Meeting on Pre Stress Concrete and Reactor Pressure Vessels and their Thermal Isolation, Brussels. pp. 237-265.

Komarovskii, A., 1961. Construction of Nuclear Installations. State Publishing House of Power Literature.

Komonen, J., Penttala, V., 2003. Effects of high temperature on the pore structure and strength of plain and polypropylene fiber reinforced cement pastes. Fire Technology 39, $23-34$.

Konings, R. (Ed.), 2012. Comprehensive Nuclear Materials. volume 1-5. Elsevier.

Kontani, O., Ichikawa, Y., Ishizawa, A., Takizawa, M., Sato, O., 2010. Irradiation effects on concrete structure, in: International Symposium on the Ageing Management and Maintenance of Nuclear Power Plants, pp. 173-182. 
Kontani, O., Sawada, S., Maruyama, I., Takizawa, M., Sato, O., 2013. Evaluation of irradiation effects on concrete structure - gamma ray irradiation tests on cement paste, in: Proceedings of the ASME 2013 Power Conference POWER2013, Boston MA, USA.

de Larrard, F., Belloc, A., 1997. The influence of aggregate on the compressive strength of normal and high-strength concrete. ACI Materials Journal 94, 417-426.

Le Pape, Y., Field, K., Remec, I., 2014. Perspective on radiation effects in concrete for nuclear power plants - part ii: Perspective from micromechanical modeling. Submitted to Journal of Nuclear Engineering and Design .

Łowińska-Kluge, A., Piszora, P., 2008. Effect of gamma irradiation on cement composite observed with xrd and sem methods in the range of radiation dose 0-1409 mgy. Acta Physica Polonica 114, 399-411.

Lucius Pitkin, Inc., 2013. Expected Condition of Concrete at Age 80 Years of Reactor Operation. Technical Report A13276-R-001. Lucius Pitkin, Inc.

Luthra, J., 1969. X-ray studies on pile-irradiated calcite. Indian Journal of Pure Applied Physics , 444-445.

Lyon, R., 1951. General Reactor Engineering Research Quarterly Progress Report for Period Ending November 20, 1950. Technical Report ORNL-973. Oak Ridge National Laboratory.

Maerker, R., 1987. Lepricon analysis of the pressure vessel surveillance dosimetry inserted into h. b. robinson-2 during cycle 9. Nuclear Science and Engineering 96, 263-289.

Maruyama, I., Kontani, O., Sawada, S., Sato, O., Igarashi, G., Takizawa, M., 2013. Evaluation of irradiation effects on concrete structure - background and preparation of neutron irradiation test, in: Proceedings of the ASME 2013 Power Conference POWER2013, Boston MA, USA.

Maruyama, I., Sasano, H., Nishioka, Y., Igarashi, G., 2014. Strength and young's modulus change in concrete due to long-term drying and heating up to $90{ }^{\circ} \mathrm{c}$. Cement and Concrete Research 66, 48-63.

Mayer, G., Lecomte, M., 1960. Effect of fast neutrons on crystalline quartz and vitreous silica. Journal de Physique et le Radium 21.

Metha, P., Monteiro, P., 2005. Concrete: Microstructrue, Properties, and Materials. 3 ed., McGraw-Hill Professional.

Naus, D., 2005. The effect of elevated temperature on concrete materials and structures A literature review. Technical Report ORNL/TM-2005/553. Oak Ridge National Laboratory.

Naus, D., 2010. A Compilation of Elevated Temperature Concrete Material Property Data and Information for Use in Assessments of Nuclear Power Plant Reinforced Concrete Structures. Technical Report NUREG/CR-7031, ORNL/TM-2009/175. Oak Ridge National Laboratory.

Naus, D., 2012. Concrete, in: Konings, R. (Ed.), Comprehensive Nuclear Materials. Elsevier. volume 4 . 
Neville, A., 1963. Properties of Concrete. John Wiley and Sons, Ltd.

ORNL, 1997. One-, two- and three-dimensional discrete ordinates neutron/photon transport code system, rsicc computer code collection package ccc-650. URL: https://rsicc.ornl.gov/codes/ccc/ccc6/ccc-650.html.

Pederson, A., 1971. Radiation damage in concrete - measurements on miniature specimens of cement mortar, in: Benzler, H. (Ed.), Proceedings of an Information Exchange Meeting on 'Results of Concrete Irradiation Programmes', Commission of the European Communities, Brussels, Belgium.

Pihlajavaara, S., 1974. A review of some of the main results of a research on the ageing phenomena of concrete: Effect of moisture conditions on strength, shrinkage and creep of mature concrete. Cement and Concrete Research 4, $761-771$.

Price, B., Horton, C., Spinney, K., 1957. Radiation Shielding. Pergamon Press.

Primack, W., 1958. Fast-neutron-induced changes in quartz and viterous silica. Physical Review 110, 1240-1254.

Rappeneau, J., Lagorio, M., Gilbert, J., Piron, P., 1966. Irradiation tests of concretes. Bulletin d'Information Scientifique et Technique 110, 31-48.

Remec, I., 1999. Study of the Neutron Flux and Dpa Attenuation in the Reactor PressureVessel Wall. Technical Report ORNL/NRC/LTR-99/5. Oak Ridge National Laboratory.

Remec, I., Kam, F., 1997. H.B. Robinson-2 Pressure Vessel Benchmark. Technical Report ORNL/TM-13204. Oak Ridge National Laboratory.

Rhoades, W., 1997. The TORT Three-Dimensional Discrete Ordinates Neutron/Photon Transport Code (TORT Version 3). Technical Report ORNL/TM13221. Oak Ridge National Laboratory.

Risner, J., Wiarda, D., Dunn, M., Miller, T., Peplow, D., Patton, B., 2011. Production and Testing of the VITAMIN-B7 Fine-Group and BUGLE-B7 Broad-Group Coupled Neutron/Gamma Cross-Section Libraries Derived from ENDF/B-VII.0 Nuclear Data. Technical Report ORNL/TM-2011/12. Oak Ridge National Laboratory.

Rockwell, T., 1948. Physical Tests of Core Drilling from the ORNL Graphite Reactor Shield. Technical Report ORNL-248. Oak Ridge National Laboratory.

Rockwell, T., 1956. Reactor Shielding Design Manual. McGraw-Hill Book Company, Inc.

Van de Schaaf, C., 1967. Effect of heating and radiation on some properties of mortar and concrete specimens with different compositions, in: Fernet, P. (Ed.), Information meeting on work relating to prestressed concrete vessels and their isolation, Commission of the European Communities, Bruxelles, Belgium. pp. 44-77.

Van de Schaaf, C., 1969. Ii. invloed van bestraling en verhitting op de strekte van mortels en beton, in: Second information meeting on prestressed concrete reactor pressure vessels and their thermal isolation, Commissions of the European Communities, Bruxelles, Belgium. pp. $172-178$. 
Schneider, U., 1981. Behavior of Concrete at High Temperature. Wilhelm Ernst and Son.

Seeberger, J., Hilsdorf, H., 1982. Einfluß von radioactiver Strahlung auf die Festogkeit and Struktur von Beton. Technical Report NR 2505. Institut für Massivbau and Baustofftechnologie, Universität Karlsruhe.

Sommers, J., 1969. Gamma irradiation damage of structural concrete immersed in water. Health Physics 16, 503-508.

Soo, P., Milian, L., 2001. The effect of gamma radiation on the strength of portland cement mortars. Journal OF Materials Science Letters 20, 1345-1348.

Stoces, B., Otopal, P., Juricka, V., Gabriel, J., 1970. The Effect of Radiation on the Mechanical Properties of Concrete. Technical Report UJV 2390-RT. UJV.

Swamy, R. (Ed.), 1996. The Alkali-Silica Reaction in Concrete. Blackie and Son Ltd.

Templier, C., Desage, F., Desoyer, J., Hishmeh, G., Cartz, L., Donnelly, S., Vinyakov, V., Birtcher, R., 1996. Ion beam amorphization of muscovite mica. Journal of Materials Research 11, 1819-1824.

U.S. Bureau of Reclamation, 1988. Concrete Manual. 8 ed. U.S. Bureau of Reclamation.

Wang, S., Wang, L., Ewing, R., 2000. Irradiation-induced amorphization: Effects of temperature, ion mass, cascade size, and dose rate. Physical Review B 63.

Was, G., 1997. Fundamentals of Radiation Materials Science. Springer.

Weber, W., Ewing, R., Catlow, C., de la Rubia, T., Hobbs, L., Kinoshita, C., Matzke, H., Motta, A., Nastasi, M., Salje, E., Vance, E., Zinkle, S., 1998. Radiation effects in crystalline ceramics for the immobilization of high-level nuclear waste and plutonium. JOURNAL OF MATERIALS RESEARCH 13, 1434-1484. doi:10.1557/JMR.1998.0205.

Weissmann, S., Nakajima, K., 1963. Defect structure and density decrease in neutronirradiated quartz. Journal of Applied Physics 34, 611-618.

White, J., Ingersoll, D., Wright, R., Hunter, H., Slater, C., Greene, N., MacFarlane, R., Roussin, R., 1994. Production and Testing of the VITAMIN-B6 fine-group and the BUGLE-93 Broad-Group Neutron/Photon Cross-section Libraries Derived from ENDF/B-VI Nuclear Data. Technical Report NUREG/CR-6214, Rev. 1. U.S. Nuclear Regulatory Commission.

Wittels, M., Sherrill, F., 1954. Radiation Damage in SiO2 Structures. Physical Review 93, $1117-1118$.

Wong, C., 1974. Neutron radiation damage in some birefringent crystals. Physics Letters $50 \mathrm{~A}, 346$.

Xiao, J., Konig, G., 2004. Study of concrete at high temperature in china-an overview. Fire Safety Journal 39, 89-103.

Zhang, B., Bicanic, N., Pearce, C., Phillips, D., 2002. Relationship between brittleness and moisture loss of concrete exposed to high temperatures. Cement and Concrete Research $32,363-371$. 
Zinkle, S., Kinoshite, C., 1997. Defect production in ceramics. Journal of Nuclear Materials 251, 200-217.

Zubov, V., Ivanov, A., 1966. Expansion of quartz caused by irradiation with fast neutrons. Soviet Physics Crystallography 11, 372. 